\title{
Computability and Adaptivity in CFD
}

\begin{abstract}
We give a brief introduction to research on adaptive computational methods for laminar compressible and incompressible flow, and then focus on computability and adaptivity for turbulent incompressible flow, where we present a framework for adaptive finite element methods with dualitybased a posteriori error control for chosen output quantities of interest. We show in concrete examples that outputs such as mean values in time of drag and lift of a bluff body in a turbulent flow are computable to a tolerance of a few percent, for a simple geometry using some hundred thousand mesh points, and for complex geometries some million mesh points.
\end{abstract}




\title{
Computability and Adaptivity in CFD
}

\author{
Johan Hoffman ${ }^{1,2}$, Johan Jansson ${ }^{2,1}$, Niclas Jansson ${ }^{1}$, \\ Rodrigo Vilela De Abreu ${ }^{1}$ and Claes Johnson ${ }^{1}$ \\ ${ }^{1}$ KTH Royal Institute of Technology, Stockholm, Sweden \\ ${ }^{2}$ Basque Center for Applied Mathematics, Bilbao, Spain
}

\section{Introduction}

The Navier Stokes (NS) equations form the basic mathematical model of fluid mechanics, used extensively e.g. in hydro- and aerodynamics, processing industry, biology, oceanography, meteorology, geophysics and astrophysics, capable to model incompressible and compressible flow, Newtonian and non-Newtonian fluids, viscous laminar flow, and turbulent flow dominated by inertial effects with chaotic particle trajectories and vortices on a range of scales in space and time. Computational fluid dynamics (CFD) concerns the digital/computational simulation of fluid flow by solving the NS equations numerically. In this chapter we consider the following aspects of CFD: (i) computability of solutions to the NS equations, intimately connected to the mathematical problem of error estimation, and (ii) adaptive algorithms for efficient computation of such solutions, a very active research area with many important contributions. This chapter is not an exhaustive review of the research area, instead we focus on (i)-(ii) for high Reynolds number turbulent flow, a topic that is not covered in other chapters of the Encyclopedia, and that we believe will be of key interest in the coming years in a multitude of areas, e.g. in vehicle aerodynamics, ocean and atmosphere sciences, and for renewable energy technologies.

The Reynolds number $R e=U L / \nu$ is used to characterise different flow regimes, where $U$ is a characteristic flow velocity, $L$ a characteristic length scale, and $\nu$ the kinematic viscosity of the fluid. For low Reynolds number (less than $R e \approx 1$ ) we have creeping flow where viscous effects are completely dominating the non-linear inertial effects. If the Reynolds number is increased the creeping flow develops into laminar flow with nonlinear features such as recirculation zones, and under further increase of the Reynolds number the flow becomes unsteady $(R e \approx 100)$, and then undergoes transition to turbulence in wakes $(R e \approx 1000)$, shear layers $\left(R e \approx 10^{4}\right)$ and boundary layers $\left(R e \approx 10^{5}\right)$. Turbulent flow shows features on a range of length scales down to a smallest scale (the Kolmogorov microscale) of size $R e^{-3 / 4}$, assuming $L=1$, where kinetic energy is dissipated to heat. At high Reynolds number turbulent flow the larger scales are purely governed by inertial effects, and consequently shear stresses can effectively be neglected at the macroscopic scales, which simplifies 
constitutive models. On the other hand, in many applications of scientific and industrial interest $R e$ is very large, of the order $10^{6}$ or larger, and the flow shows a combination of laminar and turbulent features. To accurately resolve all the features of a turbulent flow at $R e=10^{6}$ in a direct numerical simulation (DNS) would require of the order $R e^{3}=10^{18}$ mesh points in space-time, which today is impossible on any foreseeable computer. To overcome the impossibility of DNS at high Reynolds numbers, various techniques of turbulence modeling have been proposed to take into account the unresolved turbulent scales, such as RANS models based on statistical ensemble averaging and LES (subgrid) models based on spatial filtering. A common drawback of turbulence models is the unknown model parameters that need to be calibrated to the particular CFD problem at hand.

Thus, to simulate the macroscopic properties of turbulent flow is simple in the sense that constitutive models involving the effect of shear stresses can be neglected, but on the other hand it is hard due to the inability to resolve all scales in a DNS which introduces new model parameters in turbulence (subgrid) models. A rigorous error analysis of turbulence models is challenging, since averaging (filtering) of the nonlinear NS equations introduces a model error in terms of unresolved scales, the so called Reynolds (subgrid) stresses, which must be expressed in terms of the resolved scales (the closure problem). Alternatively, the model error estimation problem can be posed as an Uncertainty Quantification (UQ) problem, where the effect of the uncertain model is estimated using statistical methods, see e.g. [1, 2, 3]. Turbulence models are not the focus of this chapter, but are discussed in detail in other chapters of the Encyclopedia.

\subsection{Adaptive methods in CFD}

In an adaptive algorithm, the computational method is optimized during the computation based on local error indicators until a certain global stopping criterion is satisfied. Error indicators and stopping criterions may be constructed ad hoc based on solution features, or based on estimates of local truncation errors; see e.g. [4]. In this chapter we focus on adaptive methods based on a posteriori error estimation of the global error, which is directly related to computability of solutions to the underlying partial differential equations. A posteriori error estimation is traditionally done with respect to an energy-norm, naturally induced by the underlying differential operator, resulting in estimates in terms of computable residuals measuring the local error; see e.g. [5, 6, 7]. For surveys and references on this approach we refer to $[8,9]$.

Unfortunately, in most applications the energy-norm does not provide useful bounds on the error in quantities of physical interest. Therefore, a framework for a posteriori error estimation of arbitrary functional output was developed using duality arguments, an idea previously used in the context of postprocessing quantities of physical interest in elliptic model problems; see [10]. A framework for more general problems was then systematically developed, see e.g. $[11,12,13,14,15]$. The methodology is often referred to as a dual weighted residual method (DWR) or goal- 
oriented error estimation. For a detailed account of the development and application of this framework we refer in particular to the review papers $[16,17,18]$. In CFD, applications of adaptive finite element methods based on this framework have been increasingly advanced, for laminar incompressible flow, see e.g. [19, 20, 21, 22, 23], and for laminar compressible flow, see e.g. $[24,25,26,27,28,29,30,31]$.

Classical adaptive finite element methods concern adaptive refinement or coarsening of the computational mesh, referred to as $h$-adaptive methods, but adaptive choice of the approximation order of the method is also possible, so called $p$-adaptive methods, see e.g. [32], with also the combination of the two; hp-adaptive methods, see e.g. [33, 34, 35]. Smoothing or optimisation of the mesh without changing the mesh topology is referred to as r-adaptivity, and anisotropic mesh adaptation aligned to local flow features is another recent focus in the area, see e.g. [36, 37, 38, 39, $40,41,42,43]$.

\subsection{Computability of turbulent flow}

The challenge of computing turbulent flow is twofold: first the range of turbulent scales make full resolution impractical, and second, turbulent flow is chaotic which limits predictability due to high sensitivity to discretisation errors from the numerical method, uncertainties in data, and model errors in the case of turbulence models. The basic question of computability/predictability for a given flow may be formulated as follows: what quantity can be computed/predicted to what tolerance to what cost? Here the quantitative aspects are emphasised, both concerning the choice of quantity of interest, or output, the error tolerance, and the cost. The cost reflects the computational work and the required precision in data. We expect a turbulent flow to be more expensive to compute than a laminar flow, and a pointwise quantity (e.g. the viscous stresses at specific points) to be more expensive than an average quantity (e.g. the drag or lift of an airplane), and of course we expect the cost to increase with a decreasing tolerance.

Adaptive methods for turbulent flow have great potential; turbulent flow structures are typically concentrated to certain parts of the computational domain, which thus require high resolution, whereas in other parts of the domain the flow is laminar and can be left with low resolution. Without prior knowledge of the flow the optimal mesh resolution must be adaptively constructed based on approximate computational solutions, e.g. through a posteriori error estimation.

A posteriori error estimation for DNS connects to a stability analysis of the transient NS equations, with potentially extreme perturbation growth due to the chaotic nature of turbulent flow indicated by worst case analytical estimates. Exponential growth of the adjoint solution is reported in [44] for computing drag of a cylinder at $R e=500$, which conforms with such worst case estimates. This is contrary to observations made for bluff body flow at higher Reynolds numbers, reported e.g. in [45] for circular cylinder flow at $R e=3900$, where the adjoint solution is stable in time and under mesh refinement. The reason for these conflicting findings is still an open problem; is it related to differences in the computational 
methods used to compute approximate adjoint solutions, or is it related to a fundamental difference between to two flows? The cases $R e=500$ and $R e=3900$ show important differences; flow at $R e=500$ shows a more pronounced von Karman street, whereas the turbulent wake is more stable at $R e=3900$. Also, blow up in the adjoint solution is observed in the case of 2D compressible flow at increasing Reynolds numbers [46], where strong vorticity production in the primal problem is the result of the sharpening of boundary layers, without any non-viscous mechanism for dissipation of vorticity available in $2 \mathrm{D}$. In $3 \mathrm{D}$, turbulence develop that dissipate vorticity through vortex stretching, which can be observed in the corresponding 3D simulations [45]. In [47] we argue that the stability of the adjoint problem is possible due to cancellation, where exponential growth is alternating with exponential decay. In particular, a turbulent wake is dominated by vortices on a range of scales where each individual vortex tube is a stable flow structure in the sense that a linearised stability analysis show no exponential perturbation growth.

The extension of the framework for a posteriori error estimation to turbulence models is not obvious. For example, even though the framework can be applied to RANS models to control the discretisation error, see e.g. [48], to estimate the model error introduced by the turbulence model the closure problem must be confronted. In $[49,50]$, the closure problem of LES was made explicit in a posteriori error analysis of the filtered NS solution, taking into consideration both the discretization error of the filtered NS equations and the model error from unresolved subgrid scales, with separate discretization and model residuals. Typically, the model residual, connected to the Reynolds subgrid stress term, is hard or impossible to estimate without any prior knowledge. In [49] the model residual was estimated through scale extrapolation in a Haar basis by an assumption of scale similarity; an approach developed for convectiondiffusion-reaction equations in $[51,52,53]$. Although the method showed promising results, for general meshes this approach may become overly complex. In [54] the authors used scale extrapolation to seek to balance the relative size of discretisation and model errors by varying discretisation and model parameters. For an overview of different approaches to error assessment in turbulence, see e.g. [55, 56].

\subsection{Direct Finite Element Simulation of Turbu- lence}

The separation into numerical errors and model errors can also be questioned. Since the beginning of CFD, numerical stabilization and turbulence modelling have been intertwined, e.g. through the von NeumannRichtmyer artificial viscosity [57] and the classical Smagorinsky subgrid model [58], or more recently through the Variational Multiscale Method (VMM) for turbulence modeling [59], which connects a family of residualbased subgrid models to finite element stabilization techniques. Implicit LES (ILES) [60] is based on the idea that the dissipation from the numer-

ical method can be used to model the effect of unresolved turbulent scales without any additional explicit subgrid model, an idea which we also find 
in [50], there referred to as underresolved DNS. Whether or not a physics based explicit turbulence (subgrid) model is needed to simulate turbulent flow has occasionally formed a sharp division in the CFD community.

Residual-based numerical stabilization holds the attractive feature of being consistent, so that an exact solution of the Navier-Stokes equations satisfies also the stabilized finite element formulation of the equations. Similarly, with a residual-based subgrid model it is possible to directly connect the finite element approximation to weak solutions of the NavierStokes equations, which provides a mathematical framework for computational approximation of mean values in turbulent flow, in the form of functionals of weak solutions, without introducing LES filtering or RANS statistical averaging, and thus circumventing the closure problem. Combined with duality based a posteriori estimation of the error in such functionals of weak solutions, adaptive methods for turbulent flow have been developed under the notions of Adaptive DNS/LES, General Galerkin (G2), and Direct Finite Element Simulation (DFS) [61, 47, 62, 45, 63, 64, 65, 66]. In particular, it is shown that the adjoint solution may be computed at an affordable cost for complex turbulent flows in 3D. The method may be viewed as producing an approximate weak solution of the NS equations, with a residual that in a weak sense is small (reflecting the Galerkin orthogonality) and which is also controlled in a strong sense (reflecting the residual-based stabilization) [67, 68, 69].

In this chapter we will review this framework for direct finite element simulation of turbulence, with in particular a computational study of a model problem, and we will give examples of applications of the framework to complex engineering problems.

\section{The Navier-Stokes equations}

The NS equations were formulated in 1821-1845 and appear to give an accurate description of a great variety of fluid flows, including both laminar flow with ordered flow features and turbulent flow with vortices on a range of scales in a complex interaction. Even though the NS equations have been known for almost 200 years a basic mathematical understanding of the equations is missing, in particular the basic questions of existence and uniqueness of solutions stand without a clear answer.

The incompressible NS equations for a unit density Newtonian fluid with constant kinematic viscosity $\nu>0$ enclosed in a volume $\Omega$ in $\mathbb{R}^{3}$ (where we assume that $\Omega$ is a polygonal domain), together with suitable boundary conditions, take the form:

$$
R(\hat{u})=0, \quad \text { in } \Omega \times I
$$

for $\hat{u}=(u, p)$, with $u(x, t)$ the velocity vector and $p(x, t)$ the pressure at $(x, t), I=(0, T)$ is a time interval, and $R(\hat{u}) \equiv \bar{R}(\hat{u})-(f, 0)=$ $\left(\bar{R}_{1}(\hat{u}), \bar{R}_{2}(u)\right)-(f, 0)$, with 


$$
\begin{aligned}
& \bar{R}_{1}(\hat{u})=\dot{u}+u \cdot \nabla u+\nabla p-\nu \Delta u \\
& \bar{R}_{2}(u)=\nabla \cdot u
\end{aligned}
$$

The main existence result available is due to Jean Leray, who in 1934 proved the existence of a so-called weak solution (or turbulent solution in the terminology of Leray) [70], which is a solution satisfying (1) in an average sense; $\hat{u}$ is a weak solution if

$$
((R(\hat{u}), \hat{v}))=0
$$

for all test functions $\hat{v}$ in a test space $\hat{V}$ with norm $\|\cdot\|_{\hat{V}}$, consisting of suitable differentiable functions, $R(\hat{u})$ is assumed to belong to a space dual to $\hat{V}$, and $((\cdot, \cdot))$ denotes a duality pairing.

An idea in contemporary mathematics is now to try to extend the result of Leray, to prove existence and uniqueness of a strong solution $\hat{u}$, that would make the residual pointwise zero; $R(\hat{u})=0$. The idea, expressed in the Clay Institute $\$ 1$ million Prize Problem would be to prove that in fact Leray's weak solution $\hat{u}$ is a strong solution, by proving regularity of $\hat{u}$. With a pointwise bound on first-order derivatives, uniqueness would then follow from a standard Grönwall estimate, bounding the pointwise difference between two strong solutions in terms of a small perturbation in data, which one then would let go to zero, which would give uniqueness.

Although, the use of a Grönwall estimate would bring in an exponential factor $e^{K T}$, with $K$ a pointwise bound of the gradient of the velocity, which may be estimated to be of the order $K \sim R e^{1 / 2}$ in a turbulent flow. For $R e=10^{6}$ and $T=10$, this would result in the enormous factor $e^{10000}$, which is much larger than $10^{100}$, a googol. Any uniqueness proof involving such constants seems to lack any practical meaning, since virtually anything could then result from almost nothing.

The problem of proving uniqueness should come as no surprise, since in a turbulent flow we may expect an extreme pointwise perturbation growth, and thus pointwise uniqueness is too much to ask for. On the other hand, some aspects of turbulent flow are more well determined. Typically we expect various types of mean value output in a turbulent flow to be well determined to a higher degree than pointwise output. Uniqueness in output of a solution is intimately connected to predictability/computability. A basic question is then:

What outputs of a turbulent flow are computable to what tolerance to what cost?

In a turbulent flow we do not expect point values to be predictable/computable to any tolerance of interest, but we expect that certain mean values can be predicted/computed to a tolerance of interest. We may compare to weather prediction, which concerns solving equations of NS type for the atmosphere: in January we may expect to be able to predict the mean temperature in London for the month of June to a tolerance of say $\pm 2^{\circ} \mathrm{C}$, but we do not expect to be able to predict the daily temperature in London June 20th to a tolerance of less than say $\pm 10^{\circ} \mathrm{C}$, which is not very useful. For the problem of computing the mean drag of bluff bodies 
in a turbulent flow, a tolerance of a few percent seems reasonable, based on computational and experimental experience, but we cannot expect to be able to predict drag to an arbitrary small tolerance.

In [47] we presented a novel approach to assess the uniqueness and computability of various mean value output in turbulent flow, where we relax Leray's weak solution concept, to the concept of an $\epsilon$-weak solution, where we say that $\hat{u}$ is an $\epsilon$-weak solution if

$$
|((R(\hat{u}), \hat{v}))| \leq \epsilon\|\hat{v}\|_{\hat{V}}, \quad \forall \hat{v} \in \hat{V}
$$

with $\epsilon$ a (small) positive number. This means that for an $\epsilon$-weak solution $\hat{u}$, we require the residual $R(\hat{u})$ to be smaller than $\epsilon$ in a weak norm dual to the strong norm of $\hat{V}$, and we denote the space of $\epsilon$-weak solutions $W_{\epsilon}$. We note that this approach is in direct contrast to the approach taken in the formulation of the Clay Prize, seeking a proof that Leray's weak solution is a strong solution; here we relax the requirements on the solution, and choosing $\epsilon=0$ would then bring us back to Leray's weak solution concept.

We show that for two $\epsilon$-weak solutions $\hat{u}, \hat{w} \in W_{\epsilon}$, we have that

$$
|M(\hat{u})-M(\hat{w})| \leq 2 \epsilon S_{\epsilon}(\hat{\psi})
$$

where $S_{\epsilon}(\hat{\psi})$ is a stability factor characterizing the stability properties of the linear functional output $M(\cdot)$, defined by $M(\hat{w}) \equiv((\hat{w}, \hat{\psi}))$. The estimate (4) expresses output uniqueness of $\epsilon$-weak solutions in a quantitative form, where the stability factor $S_{\epsilon}(\hat{\psi})$ is determined by computational approximation of a dual problem, linearized at $\hat{u}$ and $\hat{w}$, and with data $\hat{\psi}$ connecting to the output $M(\cdot)$. The crucial fact behind the success of this approach for turbulent flow is that $S_{\epsilon}(\hat{\psi})$ turns out to be stable with respect to linearization at different elements in $W_{\epsilon}$ for mean value output $M(\cdot)$, which we show computationally.

We thus relax the solution concept twofold: (i) by using a weak solution concept, and (ii) by requiring $R(u)$ to be zero in a weak sense only approximatively. We shall see that (i) and (ii) naturally come together; asking only (i) does not make much sense. With the standard Leray weak solution, corresponding to $\epsilon=0$, the stability information in (4) would be lost, since one may argue that anything times 0 is still 0 .

Further, we find that we are able to construct $\epsilon$-weak solutions for almost any $\epsilon>0$, by stabilized Galerkin finite element methods, here referred to as General Galerkin (G2) methods. For a G2 solution $\hat{U}$ we derive a posteriori error estimates, with respect to an $\epsilon$-weak solution $\hat{u}$, of the form

$$
|M(\hat{u})-M(\hat{U})| \leq \epsilon S_{\epsilon}(\hat{\psi})+\sum_{K \in \mathcal{T}} e_{D}^{K}+e_{M}^{K}
$$

with error contributions from each element $K$ in the computational mesh $\mathcal{T}$, where $e_{D}^{K}$ is an error from the Galerkin discretization in G2, $e_{M}^{K}$ is an error contribution from the stabilization in $\mathrm{G} 2$, and $\epsilon S_{\epsilon}(\hat{\psi})$ characterizes computability of the output $M(\cdot)$ in terms of a best possible accuracy, where $\epsilon$ is given by the maximal computational resources. 
It seems reasonable to aim for an accuracy in computations similar to the one in experiments. For example, experimental measurements of the drag coefficient of a circular cylinder at $R e=3900$ is presented as $0.98 \pm$ 0.05 , where the disturbance level in the experiments results in an output error of about $5 \%$. We construct an adaptive computational method based on (5) for the approximation of the output $M(\cdot)$ to a given accuracy $T O L$, using a minimal number of degrees of freedom, by formulating the minimization problem:

Find a G2 solution $\hat{U}$, with a minimal number of degrees of freedom, such that $|M(\hat{u})-M(\hat{U})| \leq T O L$,

which we solve by using the a posteriori error estimate (5), starting from a coarse mesh and successively adding new degrees of freedom based on (5) until the output error bound is less than the given tolerance TOL. For turbulent flow we sometimes refer to this method as Adaptive DNS/LES, with parts of the flow being resolved to its physical scales in a DNS and parts of the flow being left unresolved in a LES, with the stabilization in G2 acting as a subgrid model. We also refer to the method as Direct Finite Element Simulation (DFS), emphasising that no explicit turbulence/subgrid model is used.

\section{$3 \epsilon$-Weak Solutions}

Viewing the study of the NS equations, in the general case of turbulent flow, as the study of mean value output from the equations, we do not need to worry about the possible existence of any strong solutions. Instead we focus on the existence of approximate weak solutions, and (weak) uniqueness in output of such solutions.

To study weak uniqueness of the NS equations, we let an $\epsilon$-weak solution be an approximate weak solution with a residual less than $\epsilon$ in a weak norm. That is, we define for $\hat{v}=(v, q) \in \hat{V}$,

$$
\begin{aligned}
& ((R(\hat{u}), \hat{v})) \equiv((\dot{u}, v))+((u \cdot \nabla u, v))-((\nabla \cdot v, p)) \\
& +((\nabla \cdot u, q))+((\nu \nabla u, \nabla v))-((f, v))
\end{aligned}
$$

where

$$
\hat{V}=\left\{\hat{v}=(v, q) \in\left[H^{1}(Q)\right]^{4}: v \in L_{2}\left(H_{0}^{1}(\Omega)\right)^{3}\right\}
$$

and $((\cdot, \cdot))$ is the $\left[L_{2}(Q)\right]^{m}$ inner product with $m=1,3$, or a suitable duality pairing, over the space-time domain $Q=\Omega \times I$. In order for all the terms in (6) to be defined, we ask $u \in L_{2}\left(I ; H_{0}^{1}(\Omega)^{3}\right)$, $(u \cdot \nabla) u \in L_{2}\left(I ; H^{-1}(\Omega)^{3}\right), \dot{u} \in L_{2}\left(I ; H^{-1}(\Omega)^{3}\right), p \in L_{2}\left(I ; L_{2}(\Omega)\right)$, and $f \in L_{2}\left(I ; H^{-1}(\Omega)^{3}\right)$, where $H_{0}^{1}(\Omega)$ is the standard Sobolev space of vector functions being zero on the boundary $\Gamma$ and square integrable together with their first derivatives over $\Omega$, with dual $H^{-1}(\Omega)$. As usual, $L_{2}(I ; X)$ with $X$ a Hilbert space denotes the set of functions $v: I \rightarrow X$ that are square integrable.

We now define $\hat{u} \in \hat{V}$ to be an $\epsilon$-weak solution if 


$$
|((R(\hat{u}), \hat{v}))| \leq \epsilon\|\hat{v}\|_{\hat{V}}, \quad \forall \hat{v} \in \hat{V}
$$

where $\|\cdot\|_{\hat{V}}$ denotes the $H^{1}(Q)^{4}$-norm, and we define $\hat{W}_{\epsilon}$ to be the set of $\epsilon$-weak solutions for a given $\epsilon>0$. Note that for simplicity here we require also the solution $\hat{u}$ to belong to the test space $\hat{V}$, which require more regularity than necessary; for the formulation (7) to make sense, it is sufficient that $R(\hat{u})$ belongs the dual space of $\hat{V}$. Equivalently, we may say that $\hat{u} \in \hat{V}$ is an $\epsilon$-weak solution if

$$
\|R(\hat{u})\|_{\hat{V}^{\prime}} \leq \epsilon
$$

where $\|\cdot\|_{\hat{V}^{\prime}}$ is the dual norm of $\hat{V}$. This is a weak norm measuring mean values of $R(\hat{u})$ with decreasing weight as the size of the mean value decreases. Point values are thus measured very lightly.

Formally we obtain the equation

$$
((R(\hat{u}), \hat{v}))=0
$$

by multiplying the NS equation by $\hat{v}$, that is, the momentum equation by $v$ and the incompressibility equation by $q$, and integrating in spacetime. Thus, a pointwise solution $\hat{u}$ to the NS equations would be an $\epsilon$ -weak solution for all $\epsilon \geq 0$, while an $\epsilon$-weak solution for $\epsilon>0$ may be viewed as an approximate weak solution.

Below, we show how to construct $\epsilon$-weak solutions using stabilized finite element methods, and thus existence of $\epsilon$-weak solutions for any $\epsilon>0$ is guaranteed (under a certain assumption). For a computed solution $\hat{u}$, we can determine the corresponding $\epsilon$ by evaluating the residual $R(\hat{u})$.

\section{Output Sensitivity and the Dual Prob- lem}

Suppose now the quantity of interest, or output, related to a given $\epsilon$-weak solution $\hat{u}$ is a scalar quantity of the form

$$
M(\hat{u})=((\hat{u}, \hat{\psi}))
$$

which represents a mean value in space-time, where $\hat{\psi} \in L_{2}(Q)$ is a given weight function. In typical applications the output could be a drag or lift coefficient in a bluff body problem. In this case the weight $\hat{\psi}$ is a piecewise constant in space-time. More generally, $\hat{\psi}$ may be a piecewise smooth function corresponding to a mean value output.

We now seek to estimate the difference in output between two different $\epsilon$-weak solutions $\hat{u}=(u, p)$ and $\hat{w}=(w, r)$. We thus seek to estimate a certain form of output sensitivity of the space $\hat{W}_{\epsilon}$ of $\epsilon$-weak solutions. To this end, we introduce the following linearized dual problem of finding $\hat{\varphi}=(\varphi, \theta) \in \hat{V}$, such that

$$
a(\hat{u}, \hat{w} ; \hat{v}, \hat{\varphi})=((\hat{v}, \hat{\psi})), \quad \forall \hat{v} \in \hat{V}_{0}
$$

where $\hat{V}_{0}=\{\hat{v} \in \hat{V}: v(\cdot, 0)=0\}$, and 


$$
\begin{aligned}
& a(\hat{u}, \hat{w} ; \hat{v}, \hat{\varphi}) \equiv((\dot{v}, \varphi))+((u \cdot \nabla v, \varphi))+((v \cdot \nabla w, \varphi)) \\
& +((\nabla \cdot \varphi, q))-((\nabla \cdot v, \theta))+((\nu \nabla v, \nabla \varphi))
\end{aligned}
$$

with $u$ and $w$ acting as coefficients, and $\hat{\psi}$ is given data.

This is a linear convection-diffusion-reaction problem in variational form, $u$ acting as the convection coefficient and $\nabla w$ as the reaction coefficient, and the time variable runs 'backward' in time with initial value $(\varphi(\cdot, T)=0)$ given at final time $T$ imposed by the variational formulation. The reaction coefficient $\nabla w$ may be large and highly fluctuating, and the convection velocity $u$ may also be fluctuating.

Choosing now $\hat{v}=\hat{u}-\hat{w}$ in (11), we obtain

$$
\begin{aligned}
((\hat{u}, \hat{\psi}))-((\hat{w}, \hat{\psi})) & =a(\hat{u}, \hat{w} ; \hat{u}-\hat{w}, \hat{\varphi}) \\
& =((R(\hat{u}), \hat{\varphi}))-((R(\hat{w}), \hat{\varphi}))
\end{aligned}
$$

and thus we may estimate the difference in output as follows:

$$
|M(\hat{u})-M(\hat{w})| \leq 2 \epsilon\|\hat{\varphi}\|_{\hat{V}}
$$

By defining the stability factor $S(\hat{u}, \hat{w} ; \hat{\psi})=\|\hat{\varphi}\|_{\hat{V}}$, we can write

$$
|M(\hat{u})-M(\hat{w})| \leq 2 \epsilon S(\hat{u}, \hat{w} ; \hat{\psi})
$$

and by defining

$$
S_{\epsilon}(\hat{\psi})=\sup _{\hat{u}, \hat{w} \in \hat{W}_{\epsilon}} S(\hat{u}, \hat{w} ; \hat{\psi})
$$

we get

$$
|M(\hat{u})-M(\hat{w})| \leq 2 \epsilon S_{\epsilon}(\hat{\psi})
$$

which expresses output uniqueness of $\hat{W}_{\epsilon}$.

Clearly, $S_{\epsilon}(\hat{\psi})$ is a decreasing function of $\epsilon$ and we may expect $S_{\epsilon}(\hat{\psi})$ to tend to a limit $S_{0}(\hat{\psi})$ as $\epsilon$ tends to zero. For small $\epsilon$, we thus expect to be able to approximate (15) by

$$
|M(\hat{u})-M(\hat{w})| \leq 2 \epsilon S_{0}(\hat{\psi})
$$

Depending on $\hat{\psi}$, the stability factor $S_{0}(\hat{\psi})$ may be small, medium, or large, reflecting different levels of output sensitivity, where we expect $S_{0}(\hat{\psi})$ to increase as the mean value becomes more local. Normalizing, we may expect the output $M(\hat{u}) \sim 1$, and then one would need $2 \epsilon S_{0}(\hat{\psi})<1$ in order for two $\epsilon$-weak solutions to have a similar output.

Estimating $S_{0}(\hat{\psi})$ using a standard Grönwall type estimate of the solution $\hat{\varphi}$ in terms of the data $\hat{\psi}$ would give a bound of the form $S_{0}(\hat{\psi}) \leq$ $C e^{K T}$, where $C$ is a constant and $K$ is a pointwise bound of $|\nabla w|$. In a turbulent flow with $R e=10^{6}$ we may have $K \sim 10^{3}$, and with $T=10$ such a Grönwall upper bound of $S_{0}(\hat{\psi})$ would be of the form $S_{0}(\hat{\psi}) \leq C e^{K T} \sim e^{10000}$, which is an incredibly large number. It would 

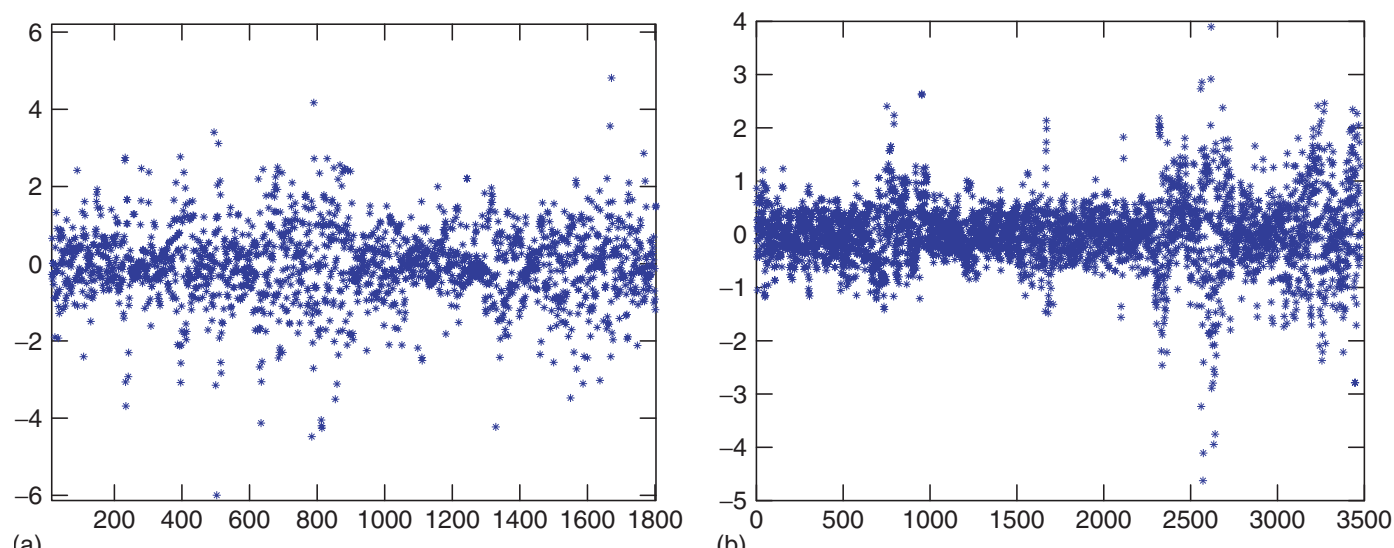

(a)

(b)

Figure 1: Sum of the real parts of the eigenvalues of $\nabla U$, the Jacobian of a G2 solution, for a few thousand elements in the turbulent wake of a circular (a) and a square cylinder (b) [47].

be inconceivable to have $\epsilon<10^{-100}$, and thus the output of an $\epsilon$-weak solution would not seem to be well defined.

However, computing the dual solution corresponding to drag and lift coefficients in turbulent flow, we find values of $S_{0}(\hat{\psi})$ that are much smaller, for which it is possible to choose $\epsilon$ so that $2 \epsilon S_{0}(\hat{\psi})<1$, with the corresponding outputs thus being well defined (up to a certain tolerance). In practice, there is a lower limit for $\epsilon$, typically given by the maximal computational resources, and thus $S_{0}(\hat{\psi})$ effectively determines the computability of different outputs.

We attribute the fact that $\hat{\varphi}$ and derivatives thereof are not exponentially large, to cancellation effects from the oscillating reaction coefficient $\nabla w$, with the sum of the real parts of the eigenvalues of $\nabla w$ being small, with the sign being about as often positive as negative; see Fig. 1. These cancellation effects appear hard to account for in purely theoretical estimates.

\section{Existence of $\epsilon$-Weak Solutions}

To generate approximate weak solutions, we use a stabilized finite element method of the form: find $\hat{U} \equiv \hat{U}_{h} \in \hat{V}_{h}$, where $\hat{V}_{h} \subset \hat{V}$ is a finite dimensional subspace defined on a computational mesh in space-time of mesh size $h$, such that

$$
((R(\hat{U}), \hat{v}))+((h R(\hat{U}), \bar{R}(\hat{v})))=0, \quad \forall \hat{v} \in \hat{V}_{h}
$$
$(w, r)$

where $R(\hat{U}) \equiv \bar{R}(\hat{U})-(f, 0)=\left(\bar{R}_{1}(\hat{U}), \bar{R}_{2}(U)\right)-(f, 0)$, and for $\hat{w}=$ 


$$
\begin{aligned}
\bar{R}_{1}(\hat{w}) & =\dot{w}+U \cdot \nabla w+\nabla r-\nu \Delta w \\
\bar{R}_{2}(w) & =\nabla \cdot w
\end{aligned}
$$

with elementwise definition of second-order terms. We here interpret a convection term $((U \cdot \nabla w, v))$ as

$$
\frac{1}{2}((U \cdot \nabla w, v))-\frac{1}{2}((U \cdot \nabla v, w))
$$

which is literally true if $\nabla \cdot U=0$. With this interpretation we will have $((U \cdot \nabla U, U))=0$, even if the divergence of the finite element velocity $U$ does not vanish exactly, and we obtain choosing $\hat{v}=\hat{U}$ in (17), and assuming that $f=0$ :

$$
\frac{1}{2}\|U\|_{\Omega}^{2}+\|\sqrt{\nu} \nabla U\|^{2}+\|\sqrt{h} R(\hat{U})\|^{2} \leq \frac{1}{2}\left\|u^{0}\right\|_{\Omega}^{2}
$$

with $\|\cdot\|_{\Omega}$ the spatial $L_{2}$-norm over $\Omega$, and $\|\cdot\|$ the space-time norm over $\Omega \times I$. The finite element method (17) is a stabilized Galerkin method with the term $((R(\hat{U}), v))$ corresponding to Galerkin's method and the term $((h R(\hat{u}), \bar{R}(\hat{v})))$ corresponding to a weighted residual least squares method with stabilizing effect expressed in (18). We also refer to this method as G2 or General Galerkin, and we thus refer to $\hat{U}$ as a G2 solution. The existence of a discrete solution $\hat{U} \equiv \hat{U}_{h} \in \hat{V}_{h}$ follows by Brouwer's fixed point theorem combined with the stability estimate in (18).

We now prove the existence of $\epsilon$-weak solutions to the NS equations for any $\epsilon>0$. For all $\hat{v} \in \hat{V}$, we have with $\pi_{h} \hat{v} \in \hat{V}_{h}$ a standard interpolant of $\hat{v}$ satisfying $\left\|h^{-1}\left(\hat{v}-\pi_{h} \hat{v}\right)\right\| \leq C_{i}\|\hat{v}\|_{\hat{V}}$, using also (17),

$$
\begin{aligned}
&|((R(\hat{U}), \hat{v}))|= \mid\left(\left(R(\hat{U}), \hat{v}-\pi_{h} \hat{v}\right)\right)-\left(\left(h R(\hat{U}), \bar{R}\left(\pi_{h} \hat{v}\right)\right) \mid\right. \\
& \leq C_{i}\|h R(\hat{U})\|\|\hat{v}\|_{\hat{V}}+M(U)\|h R(\hat{U})\|\|\hat{v}\|_{\hat{V}}
\end{aligned}
$$

where $M(U)$ is a pointwise bound of the velocity $U(x, t)$, and $C_{i} \approx 1$ is an interpolation constant. It follows that the G2-solution $\hat{U}$ is an $\epsilon$ -weak solution with

$$
\epsilon=\left(C_{i}+M(U)\right)\|h R(\hat{U})\| \leq \sqrt{h}\left(C_{i}+M(U)\right)\left\|u^{0}\right\|_{\Omega}
$$

since from the energy stability estimate (18) we have that $\|\sqrt{h} R(\hat{U})\| \leq$ $\left\|u^{0}\right\|_{\Omega}$.

Assuming now that $M(U)=M\left(U_{h}\right)$ is bounded with $h>0$, and letting $\left(C_{i}+M(U)\right)\left\|u^{0}\right\|_{\Omega} \leq C$, it follows that $\hat{U}$ is an $\epsilon$-weak solution with $\epsilon=C \sqrt{h}$. More generally, we may say that a G2 solution $\hat{U}$ is an $\epsilon$ -weak solution with $\epsilon=C_{U}\|h R(\hat{U})\|$, with $C_{U}=C_{i}+M(U)$.

We have now demonstrated the existence of an $\epsilon$-weak solution to the NS equations for any $\epsilon$, assuming that the maximum of the computed velocity is bounded (or grows slower than $h^{-1 / 2}$ ). More generally, we have shown that a G2 solution $\hat{U}$ is an $\epsilon$-weak solution with $\epsilon=C_{U}\|h R(\hat{U})\|$. Computing $\hat{U}$, we can compute $\epsilon=C_{U}\|h R(\hat{U})\|$ and thus determine the corresponding $\epsilon$. 


\section{Computability and a Posteriori Error Estimation}

We now let $\hat{u}$ be an $\epsilon$-weak solution of the NS equations with $\epsilon$ small, and we let $\hat{U}$ be a G2 solution, which can be viewed to be an $\epsilon_{G 2}$-weak solution, with $\epsilon_{G 2}=C_{U}\|h R(\hat{U})\|>>\epsilon$. As in (15), we get the following a posteriori error estimate for a mean value output given by a function $\hat{\psi}$ :

$$
|M(\hat{u})-M(\hat{U})| \leq\left(C_{U}\|h R(\hat{U})\|+\epsilon\right) S_{\epsilon_{G 2}}(\hat{\psi})
$$

where $S_{\epsilon_{G 2}}(\hat{\psi})$ is the corresponding stability factor defined by (14). Obviously the size of the stability factor $S_{\epsilon_{G 2}}(\hat{\psi})$ is crucial for computability: the stopping criterion is evidently (assuming $\epsilon$ small):

$$
C_{U}\|h R(\hat{U})\| S_{\epsilon_{G 2}}(\hat{\psi}) \leq T O L
$$

where $T O L>0$ is a tolerance. If $S_{\epsilon_{G 2}}(\hat{\psi})$ is too large, or TOL is too small, then we may not be able to reach the stopping criterion with available computing power, and the computability is out of reach. In applications we estimate $S_{\epsilon_{G 2}}$ by computational approximation of the dual problem.

We note that for weak uniqueness the residual only needs to be small in a weak norm, and correspondingly for computability the G2 residual only needs to be small when weighted by $h$. This means that for accurate approximation of a mean value output, the NS equations do not need to be satisfied pointwise, corresponding to a pointwise small residual, but only in an average sense, corresponding to the residual being small only in a weak norm. In computations we find that in fact the G2 residual typically is large pointwise for solutions corresponding to accurate approximation of mean value output, such as the drag of a bluff body.

\section{Direct Finite Element Simulation}

The main elements of Direct Finite Element Simulation of turbulent flow are now presented, in the form of a finite element method with residual based stabilization, quantitative a posteriori error estimation, and an adaptive algorithm.

\subsection{The Eulerian cG(1)cG(1) Method}

The $\mathrm{cG}(1) \mathrm{cG}(1)$ method is a variant of G2 using the continuous Galerkin method $\mathrm{cG}(1)$ in space and time. With $\mathrm{cG}(1)$ in time the trial functions are continuous piecewise linear and the test functions piecewise constant. $\mathrm{cG}(1)$ in space corresponds to both test functions and trial functions being continuous piecewise linear. Let $0=t_{0}<t_{1}<\ldots<t_{N}=T$ be a sequence of discrete time steps with associated time intervals $I_{n}=\left(t_{n-1}, t_{n}\right)$ of length $k_{n}=t_{n}-t_{n-1}$ and space-time slabs $S_{n}=\Omega \times I_{n}$, and let $W^{n} \subset H^{1}(\Omega)$ be a finite element space consisting of continuous piecewise linear functions on a mesh $\mathcal{T}_{n}=\{K\}$ of mesh size $h_{n}(x)$ with $W_{w}^{n}$ the functions $v \in W^{n}$ satisfying the Dirichlet boundary condition $\left.v\right|_{\partial \Omega}=w$. 
We now seek functions $\hat{U}=(U, P)$, continuous piecewise linear in space and time, and the cG(1)cG(1) method for the NS equations 01, with homogeneous Dirichlet boundary conditions reads: For $n=1, \ldots, N$, find $\left(U^{n}, P^{n}\right) \equiv\left(U\left(t_{n}\right), P\left(t_{n}\right)\right)$ with $U^{n} \in V_{0}^{n} \equiv\left[W_{0}^{n}\right]^{3}$ and $P^{n} \in W^{n}$, such that

$$
\begin{gathered}
\left(\left(U^{n}-U^{n-1}\right) k_{n}^{-1}+\bar{U}^{n} \cdot \nabla \bar{U}^{n}, v\right)+\left(2 \nu \epsilon\left(\bar{U}^{n}\right), \epsilon(v)\right)-\left(P^{n}, \nabla \cdot v\right)+\left(\nabla \cdot \bar{U}^{n}, q\right) \\
+S D_{\delta}\left(\bar{U}^{n}, P^{n} ; v, q\right)=(f, v) \quad \forall(v, q) \in V_{0}^{n} \times W^{n}
\end{gathered}
$$

where $\bar{U}^{n}=1 / 2\left(U^{n}+U^{n-1}\right)$, with the stabilizing term

$S D_{\delta}\left(\bar{U}^{n}, P^{n} ; v, q\right) \equiv\left(\delta_{1}\left(\bar{U}^{n} \cdot \nabla \bar{U}^{n}+\nabla P^{n}-f\right), \bar{U}^{n} \cdot \nabla v+\nabla q\right)+\left(\delta_{2} \nabla \cdot \bar{U}^{n}, \nabla \cdot v\right)$

where $\delta_{1}=1 / 2\left(k_{n}^{-2}+|U|^{2} h_{n}^{-2}\right)^{-1 / 2}$ in the convection dominated case $\nu<\bar{U}^{n} h_{n}$ and $\delta_{1}=\kappa_{1} h_{n}^{2}$ otherwise, $\delta_{2}=\kappa_{2} h_{n}$ if $\nu<\bar{U}^{n} h_{n}$ and $\delta_{2}=\kappa_{2} h_{n}^{2}$ otherwise, with $\kappa_{1}$ and $\kappa_{2}$ positive constants of unit size (here we have $\kappa_{1}=\kappa_{2}=1$ ), and

$$
\begin{aligned}
(v, w) & =\sum_{K \in \mathcal{T}_{n}} \int_{K} v \cdot w \mathrm{~d} x \\
(\epsilon(v), \epsilon(w)) & =\sum_{i, j=1}^{3}\left(\epsilon_{i j}(v), \epsilon_{i j}(w)\right)
\end{aligned}
$$

We note that the time step $k_{n}$ is given by the mesh size $h_{n}$, with typically

$$
k_{n} \sim \min _{x} \frac{h_{n}(x)}{\bar{U}^{n}(x)}
$$

\subsection{A Posteriori Error Estimation}

A mean value in time of the force on a body, over a time interval $I$, may be expressed as

$$
\begin{array}{r}
N(\sigma(\hat{u}))=\frac{1}{|I|} \int_{I}(\dot{u}+u \cdot \nabla u-f, \Phi)-(p, \nabla \cdot \Phi) \\
+(2 \nu \epsilon(u), \epsilon(\Phi))+(\nabla \cdot u, \Theta) \mathrm{d} t
\end{array}
$$

where $\hat{u}$ is an $\epsilon$-weak solution to the NS equations, and $\Phi$ is a function defined in the fluid volume $\Omega$ being equal to a unit vector in the direction of the force we want to compute on $\Gamma_{0}$, the surface of the body in contact with the fluid, and zero on the remaining part of the boundary $\Gamma_{1}=\partial \Omega \backslash \Gamma_{0}$. The representation (22) is independent of $\Theta$, and the particular extension of $\Phi$ away from the boundary, and we require that $\hat{\Phi}=(\Phi, \Theta) \in \hat{V}$.

We compute an approximation of the drag $N(\sigma(\hat{u}))$ from a cG(1)cG(1) solution $\hat{U}$, using the formula 


$$
\begin{aligned}
N^{h}(\sigma(\hat{U}))=\frac{1}{|I|} & \int_{I}(\dot{U}+U \cdot \nabla U-f, \Phi) \\
& -(P, \nabla \cdot \Phi)+(2 \nu \epsilon(U), \epsilon(\Phi))+(\nabla \cdot U, \Theta) \\
& +S D_{\delta}(U, P ; \Phi, \Theta) \mathrm{d} t
\end{aligned}
$$

where now $\Phi$ and $\Theta$ are finite element functions, and where $\dot{U}=$ $\left(U^{n}-U^{n-1}\right) / k_{n}$ on $I_{n}$. We note the presence of the stabilizing term $S D_{\delta}$ in (23) compared to (22), which is added in order to obtain the independence of $N^{h}(\sigma(\hat{U}))$ from the choice of $(\Phi, \Theta)$, given by (21).

Approximating $\hat{\varphi}=(\varphi, \theta)$, the exact solution to the dual problem (11), by a computed approximation $\hat{\varphi}_{h}=\left(\varphi_{h}, \theta_{h}\right)$, with the linearized convection velocity $u \approx U$, we are led to the following a posteriori error estimate for the time average over $I$ of the drag force on a body in a fluid, with respect to $\hat{u} \in W_{\epsilon}$ :

$$
\left|N(\sigma(\hat{u}))-N^{h}(\sigma(\hat{U}))\right| \approx \epsilon S_{\epsilon}(\hat{\psi})+\sum_{K \in \mathcal{T}_{n}} \mathcal{E}_{K, h}
$$

where $\hat{\psi}=(\Phi, 0)$ is the data to the dual problem defining the output $N(\sigma(\cdot)), k$ and $h$ are the time step and the local mesh size, respectively, and $\mathcal{E}_{K, h}=e_{D, h}^{K}+e_{M, h}^{K}$, with

$$
\begin{aligned}
e_{D, h}^{K}= & \frac{1}{|I|} \int_{I}\left(\left\|R_{1}(\hat{U})\right\|_{K} \times\left(C_{h} h^{2}\left\|D^{2} \varphi_{h}\right\|_{K}+C_{k} k\left\|\dot{\varphi}_{h}\right\|_{K}\right)\right. \\
& \left.+\left\|R_{2}(\hat{U})\right\|_{K} \times\left(C_{h} h^{2}\left\|D^{2} \theta_{h}\right\|_{K}+C_{k} k\left\|\dot{\theta}_{h}\right\|_{K}\right)\right) \mathrm{d} t \\
e_{M, h}^{K}= & \frac{1}{|I|} \int_{I} S D_{\delta}\left(\hat{U} ; \hat{\varphi}_{h}\right)_{K} \mathrm{~d} t
\end{aligned}
$$

where we may view $e_{D, h}^{K}$ as the error contribution from the Galerkin discretization in $\mathrm{cG}(1) \mathrm{cG}(1)$, and $e_{M, h}^{K}$ as the contribution from the stabilization in $\mathrm{cG}(1) \mathrm{cG}(1)$, on element $K$. The lower bound on the tolerance, defining computability of the output $N(\sigma(\cdot))$, is given by $\epsilon S_{\epsilon}(\hat{\psi})$. Here we think of $\epsilon$ as being small, corresponding to a maximal computational cost, so that $\epsilon S_{\epsilon}(\hat{\psi})<<\sum_{K \in \mathcal{T}_{n}} \mathcal{E}_{K, h}$.

\subsection{The Do-nothing Error Estimate and Indica- tor}

To minimize loss of sharpness, we also investigate an approach where the weak form is used directly in a posteriori error estimates, without integration by parts to the strong form, and using Cauchy-Schwarz inequality and interpolation estimates. We here refer to this direct form of a posteriori error representation as the "do-nothing" approach.

In terms of the the exact adjoint solution $\hat{\varphi}$, the output error with respect to a weak solution $\hat{u}$ can be represented as:

$$
|M(\hat{u})-M(\hat{U})|=|((R(\hat{U}), \hat{\varphi}))|=\left|\sum_{K \in \mathcal{T}_{n}}((R(\hat{U}), \hat{\varphi}))_{K}\right|
$$


This error representation involves no approximation or inequalities. We thus refer to the following error indicator based on the representation as the do-nothing error indicator:

$$
e_{N}^{K} \equiv((R(\hat{U}), \hat{\varphi}))_{K}
$$

A computable estimate and error indicator are again based on the computed approximation $\hat{\varphi}_{h}$ of the dual solution:

$$
\begin{aligned}
|M(\hat{u})-M(\hat{U})| & \approx\left|\left(\left(R(\hat{U}), \hat{\varphi}_{h}\right)\right)\right| \\
e_{N, h}^{K} & \equiv\left(\left(R(\hat{U}), \hat{\varphi}_{h}\right)\right)_{K}
\end{aligned}
$$

where we may lose reliability of the global error estimate by the Galerkin orthogonality property, which states that the $\left(\left(R(\hat{U}), \hat{\varphi}_{h}\right)\right)$ vanishes for a standard Galerkin finite element method if $\hat{\varphi}_{h}$ is chosen in the same space as the test functions. Although, in the setting of a stabilised finite element method this is not the case, as we will see below.

\subsection{Adaptive Algorithm}

We now present an algorithm for adaptive mesh refinement based on the a posteriori output error estimate (24). For simplicity, we here use the same space mesh and the same time step length for all time steps.

Given an initial coarse computational space mesh $\mathcal{T}^{0}$, start at $k=0$, then do:

1. Compute approximation of the primal problem using $\mathcal{T}^{k}$.

2. Compute approximation of the dual problem using $\mathcal{T}^{k}$.

3. If $\left|\sum_{K \in \mathcal{T}_{k}} \mathcal{E}_{K, h}^{k}\right|<$ TOL then STOP, else:

4. On the basis of the size of the local error indicator $\mathcal{E}_{K, h}^{k}$, mark a fixed fraction of the elements in $\mathcal{T}^{k}$ for refinement. Obtain a new refined mesh $\mathcal{T}^{k+1}$, using a standard algorithm for local mesh refinement.

5. Set $k=k+1$, then goto (1).

\section{Computability in a Model Problem}

Computability of turbulent flow is now investigated in a model problem; high Reynolds number flow past a cube in a channel. The high Reynolds number flow is approximated by zero viscosity in the cG(1)cG(1) model, and slip boundary conditions with zero skin friction. Thus there is no viscous dissipation in the model, effectively corresponding to the Euler equations, but the computed ( $\epsilon$-weak) solutions exhibit turbulent dissipation in the wake through the residual based stabilization of the method.

\subsection{Error Estimates at Three Levels}

We study the performance of the a posteriori error estimates for the model problem at three levels: (i) a global norm estimate which can be expected 
to overestimate the error due to the use of a global Cauchy-Schwarz inequality, (ii) a cell-local norm estimate based on local Cauchy-Schwarz inequalities that provide a robust estimate and indicator, and (iii) a "bare" do-nothing estimate and indicator expected to to reach maximum sharpness but possibly lose reliability due to higher sensitivity to the local approximation of the adjoint solution.

The error estimate (20) is based on global norms where the global stability factor $S_{\epsilon_{G 2}}(\hat{\psi})$ give an indication of the stability of the problem. However the estimate may grossly over-estimate the error, due to the use of global estimates. In the model problem computations we assume that the contribution from the $\epsilon$-weak solution is small and omit it, so that the global norm error estimate takes the following form:

$$
|M(\hat{u})-M(\hat{U})| \leq\left(C_{U}\|h R(\hat{U})\|\right) S_{\epsilon_{G 2}}(\hat{\psi})
$$

To increase sharpness we use the a posteriori error estimate based on cell-local norms in (24), where the Cauchy-Schwarz inequality is applied to the error representation for each cell in the mesh individually, which allows for cancellations between the residual and the dual weight. For simplicity, we omit the contribution from the $\epsilon$-weak solution and the contribution from the stabilization in the $S D_{\delta}$ term, and for robustness we use firstorder interpolation estimates instead of second-order estimates.

The cell-local norm error estimate and indicator for the model problem take the following form:

$$
\begin{aligned}
|M(\hat{u})-M(\hat{U})| \leq & \sum_{K \in \mathcal{T}_{n}} e_{D, h}^{K} \\
e_{D, h}^{K}= & \frac{1}{|I|} \int_{I}\left(\left\|R_{1}(\hat{U})\right\|_{K} \times\left(C_{h} h\left|\nabla \varphi_{h}\right|_{K}+C_{k} k\left|\dot{\varphi}_{h}\right|_{K}\right)\right. \\
& \left.+\left\|R_{2}(\hat{U})\right\|_{K} \times\left(C_{h} h\left\|\nabla \theta_{h}\right\|_{K}+C_{k} k\left\|\dot{\theta}_{h}\right\|_{K}\right)\right) \mathrm{d} t
\end{aligned}
$$

In the do-nothing a posteriori error estimate (28) no inequalities or approximations are used, aside from the discretization of the adjoint solution. This represents the "barest" estimate, which allows for additional cancellations: between the residual and the dual weight, and also between contributions of different signs, but where we may sacrifice reliability due to a higher sensitivity with respect to the local approximation of the adjoint solution. Again omitting the contribution from the $\epsilon$-weak solution the do-nothing error estimate and indicator for the model problem take the following form:

$$
\begin{aligned}
\left|M\left(\hat{u}_{e}\right)-M(\hat{U})\right| & \approx\left|\left(\left(R(\hat{U}), \hat{\varphi}_{h}\right)\right)\right| \\
e_{N_{h}}^{K} & \equiv\left(\left(R(\hat{U}), \hat{\varphi}_{h}\right)\right)_{K}
\end{aligned}
$$

\subsection{FEniCS Automated Implementation}

Developing multiphysics finite element methods (FEM) and scalable HPC implementations can be very challenging in terms of software complexity and performance, even more so with the addition of goal-oriented adaptive mesh refinement. To manage the complexity we have implemented 
the methods in the FEniCS automated open source software framework for solving PDE with FEM [71, 72, 73]. This allows taking the weak form of a partial differential equation (PDE) as input in near-mathematical notation and automatically generating the low-level implementation source code and auxiliary equations and quantities necessary for the adaptivity, with demonstrated optimal strong scaling up to ca. 20000 cores on a supercomputer.

To solve a PDE in weak form in the FEniCS framework, we first define the weak form in a UFL "form file", closely mapping mathematical notation. The form file is then compiled to low-level $\mathrm{C}++$ source code for assembling the local element matrix and vector with FFC. Finally we use DOLFIN-HPC to write a high-level "solver", here in $\mathrm{C}++$, which we denote Unicorn, composing the different abstractions, where a mesh is defined, the global matrix and vector are assembled by interfacing to the generated source code, the linear system is solved by an abstract parallel linear algebra interface (using PETSc as back-end by default).

Here we write our weak forms in an "equation sheet" in UFL notation, which in a concise format contains all our mathematical formulations.

We formulate the General Galerkin (G2) method for the incompressible Euler equations in UFL by a direct input of the strong and weak residuals. We can automatically derive the Jacobian in a quasi-Newton fixed-point formulation and also automatically linearize and generate the adjoint problem needed for the error estimates and adaptive error control.

The primal method in UFL notation is presented in Fig. 2, the adjoint method in Fig. 3, computation of the $H^{1}$ norm in Fig. 4 and the CauchySchwarz global norm error estimate in Fig. 5.

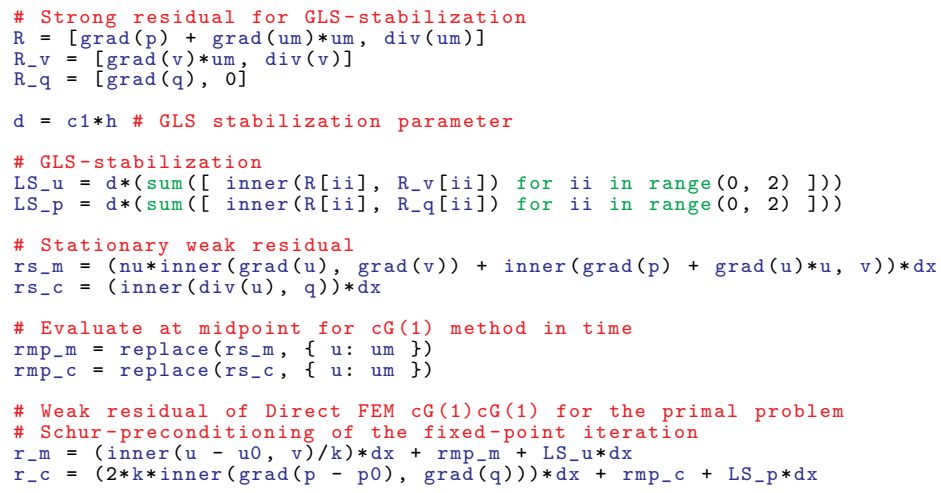

Figure 2: $c \mathrm{G}(1) \mathrm{cG}(1)$ method in FEniCS UFL notation for the primal problem, where we have omitted the declaration of the functions for brevity. 


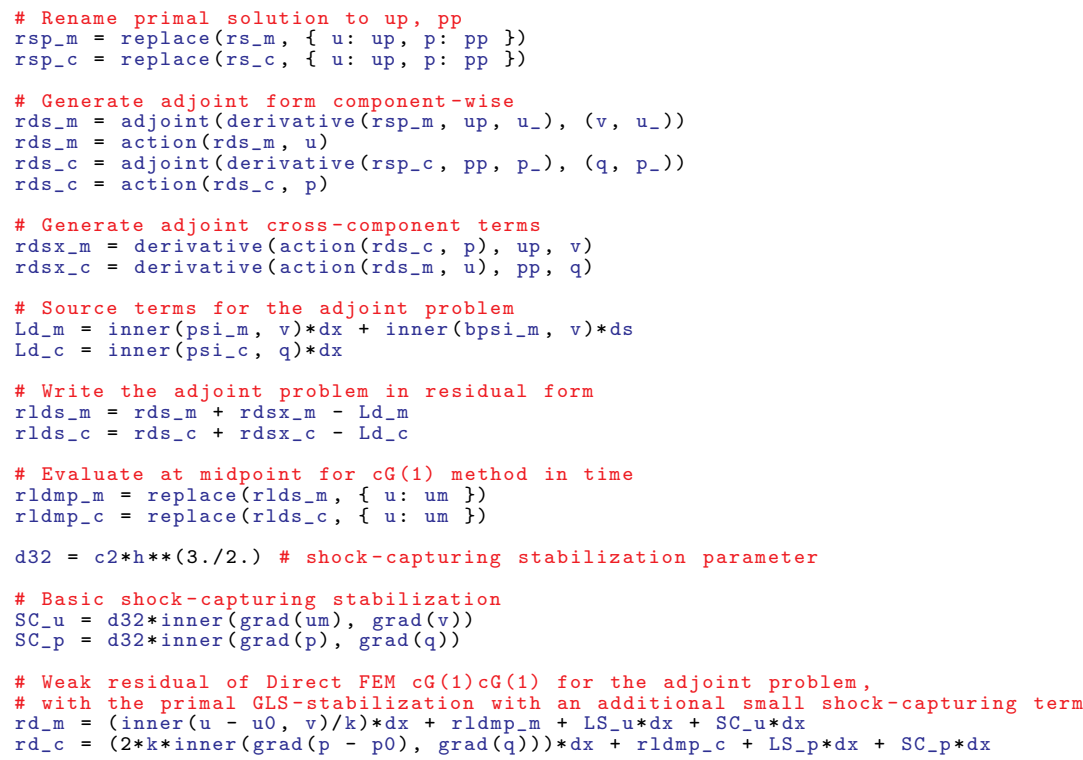

Figure 3: cG(1)cG(1) method in FEniCS UFL notation for the adjoint problem.

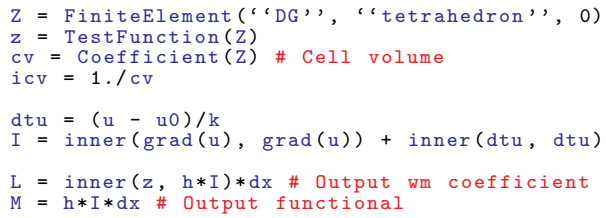

Figure 4: Computation of the square of the global $H^{1}$ seminorm of the adjoint velocity for one time step.

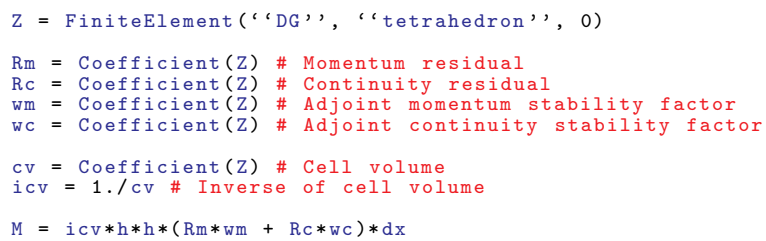

Figure 5: Computation of the square of the global Cauchy-Schwarz error estimate for one time step, with wm, wc, Rm and Rc computed as above. 


\subsection{Computability and Output Sensitivity}

We now address the question of computability in turbulence in a concrete case: more specifically computability of the drag coefficient in a DFS model of the cube model problem. We study the stability of the adjoint solution, performance of the different a posteriori error estimates, and the convergence of the drag coefficient output quantity.

The model problem is defined by a unit velocity inflow boundary condition in the $\mathrm{x}$-direction past a unit cube located at origo in a pipe in the interval $x=[-10,30]$ with radius 10 , over the time interval $\mathrm{I}=[0,400]$. The outflow boundary is modelled by a zero pressure boundary condition, and a free slip boundary condition is applied everywhere else. The cube geometry is chosen to minimize the influence of the boundary condition; the cube geometry is exactly represented by the mesh, and the sharp edges trigger flow separation so that boundary layer effects are avoided.

The initial coarse mesh has 3500 vertices, and the do-nothing adaptive method is applied to successively refine the mesh for 14 iterations. A sequence of adaptive meshes is thus generated with ca. 200k vertices in the finest mesh, see Fig. 6. For each mesh we compute the primal and the adjoint solutions, see Fig. 7 and Fig. 8.

We study the stability of the adjoint solution with respect to time and mesh refinement, i.e. the stability factor that appears in the global norm a posteriori error estimate (29). We also compute the mesh convergence of the error estimates with global and local norms, as well as the do-nothing error estimate, and compare against a reference error based on choosing the finest mesh as the truth mesh. The mesh size factor $h$ is distributed to the two factors in the error estimate, that is we study the norm $\|\sqrt{h} R(\hat{U})\|_{L_{2}(\Omega \times I)}$, and the seminorms $|\sqrt{h} \varphi|_{H^{1}(\Omega \times I)}$ and $|\sqrt{h} \theta|_{H^{1}(\Omega \times I)}$ separately.

In Fig. 10 we verify that the stability factors show only weak sensitivity to the mesh, in Fig. 11 we find that they are bounded in time, and in Fig. 12 we find that the norm of the residual decreases slowly with mesh refinement. The product of the near constant stability factor and the decreasing residual norm thus gives a converging global norm error estimate, which we verify in Fig. 13. In the same figure we also present the cell-local norm error estimate, which is sharper but still grossly over-estimates the error. Finally the do-nothing estimate in the same figure is quite sharp for this problem. The potential cancellation effects are clear visible in Fig. 9, where we plot the residual and the gradient of the adjoint solution. Convergence with respect to the drag coefficient can be seen in Fig. 13, where for the last four adaptive iterations the drag coefficient vary less than $1 \%$.

\section{Application of DFS}

The DFS methodology has been validated for a number of bluff body flow problems over a range of Reynolds numbers, and we here recall some of these studies. 

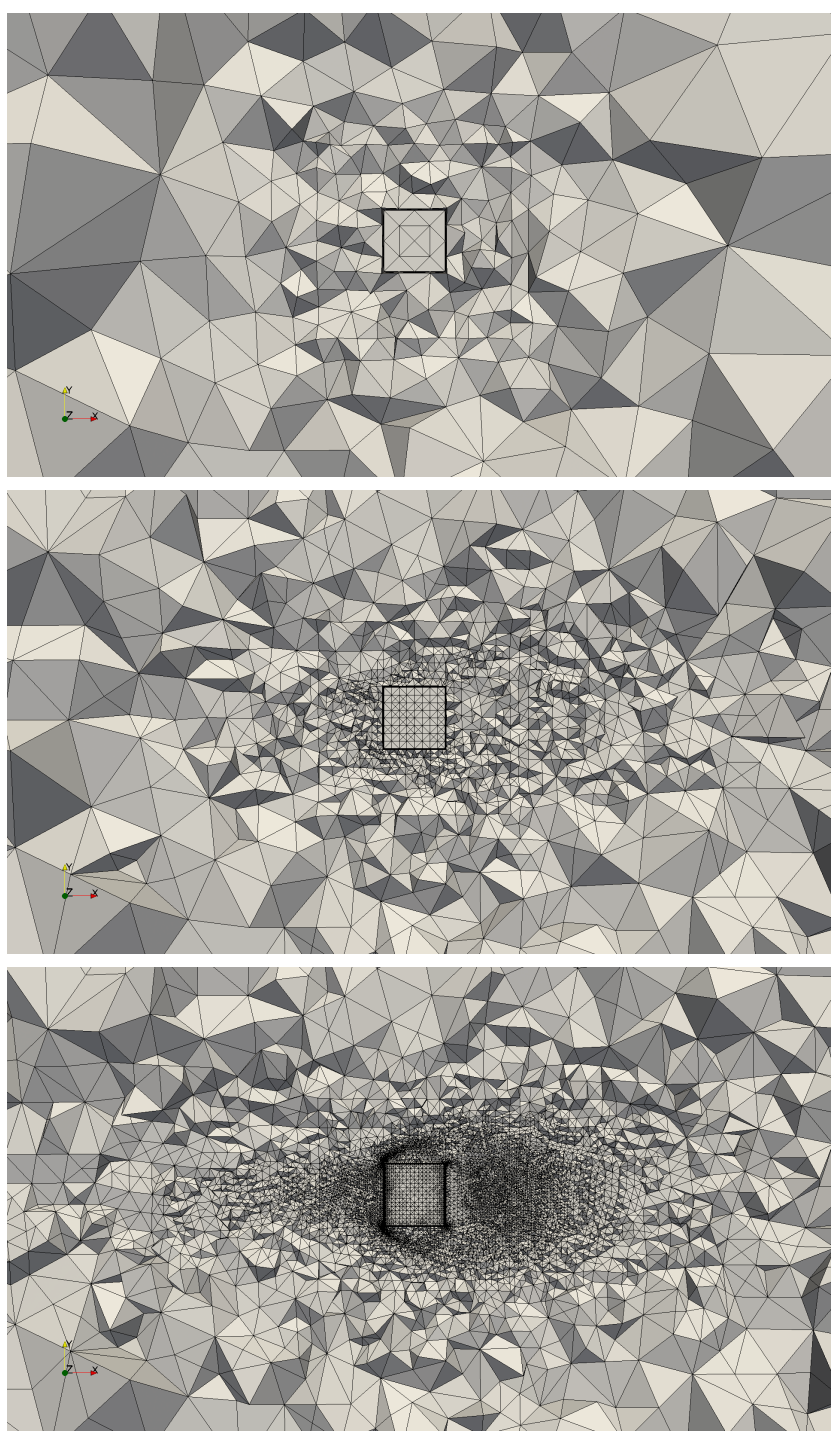

Figure 6: Adaptively refined meshes for iterations 0,5 and 15. 

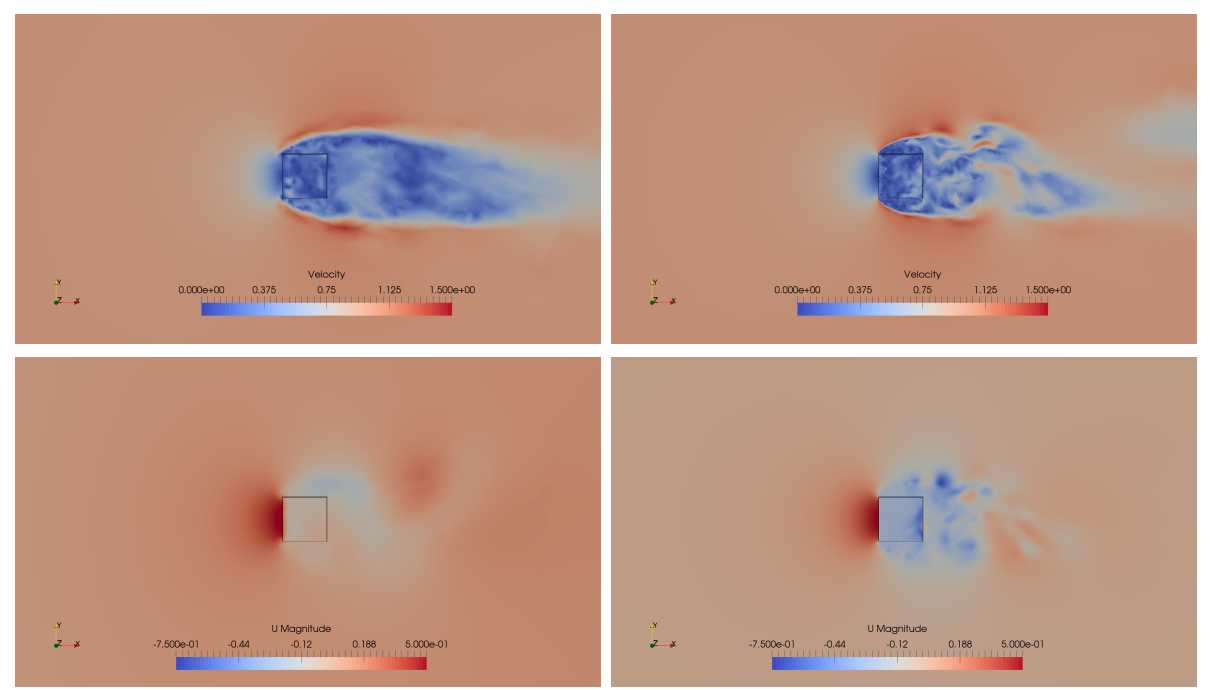

Figure 7: Snapshots of the magnitude of the primal velocity (top) and the pressure (bottom) for adaptive iterations 5 and 15.
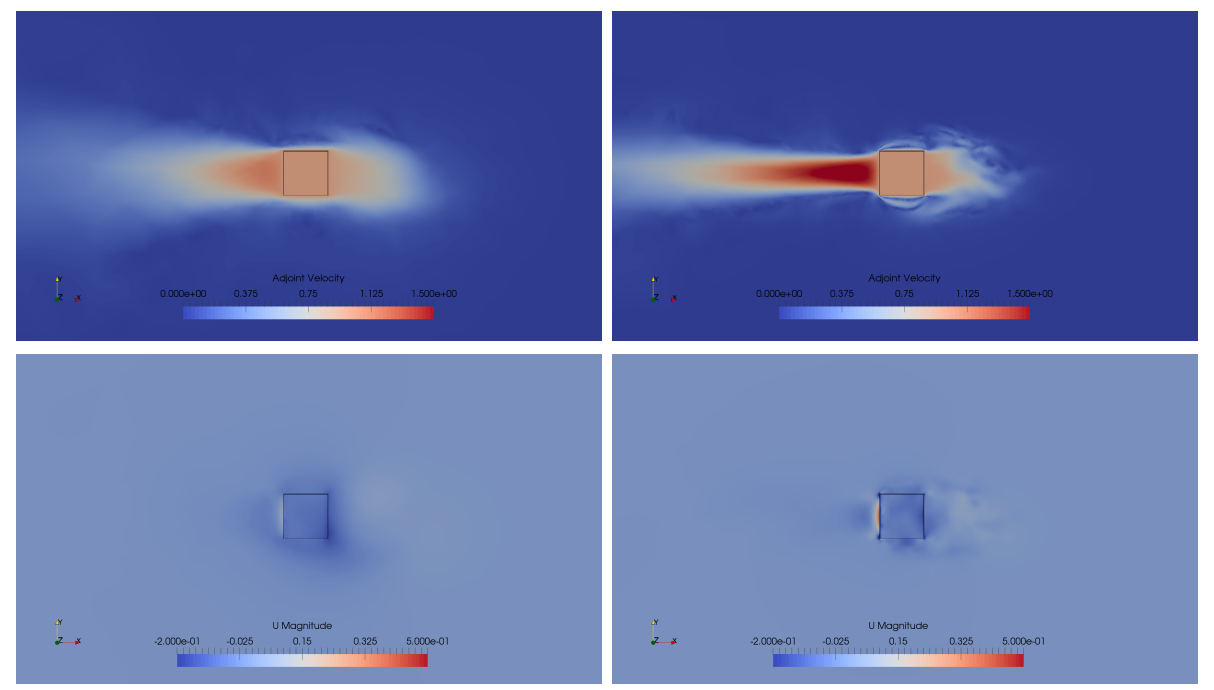

Figure 8: Snapshots of the magnitude of the adjoint velocity $\varphi_{h}$ (top) and the adjoint pressure $\theta_{h}$ (bottom) for adaptive iterations 5 and 15 

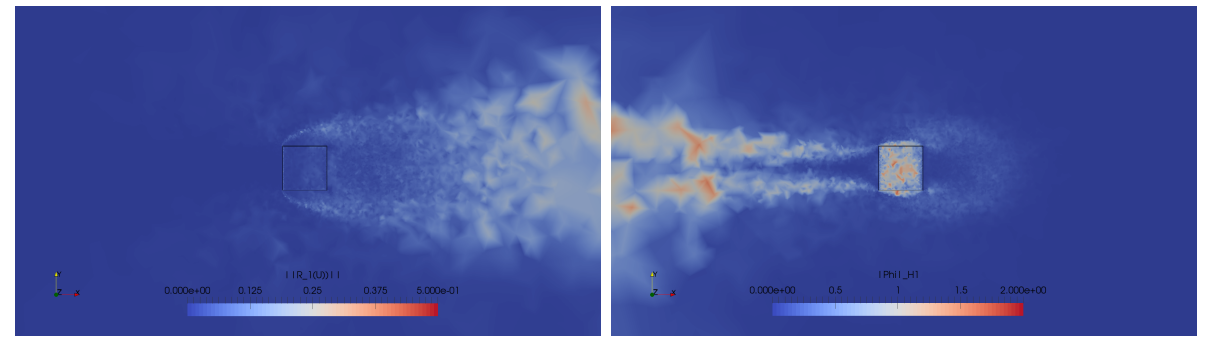

Figure 9: Snapshots of the magnitudes of the momentum residual (left) and the gradient of the adjoint velocity (right) for adaptive iteration 15 .
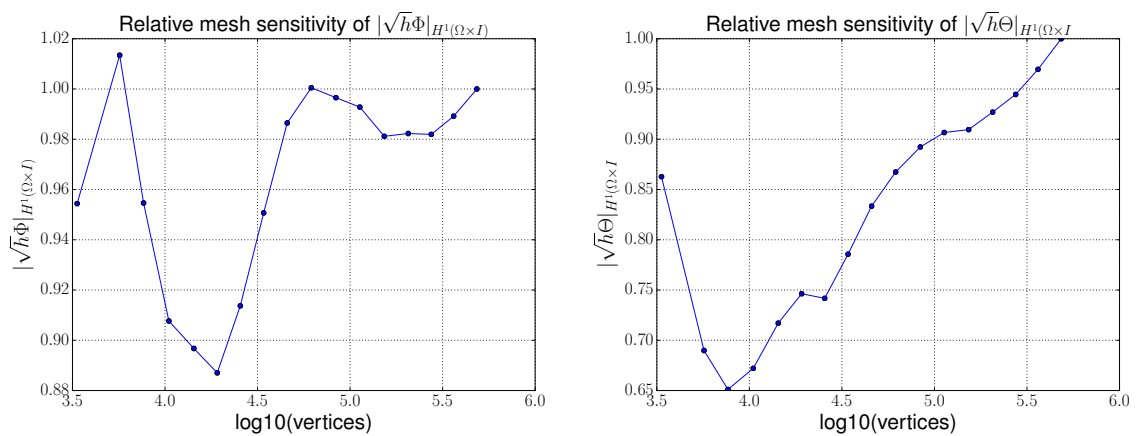

Figure 10: Mesh sensitivity of the adjoint velocity stability factor $|\sqrt{h} \varphi|_{H^{1}(\Omega \times I)}$ (left) and the adjoint pressure stability factor $|\sqrt{h} \theta|_{H^{1}(\Omega \times I)}$ (right).
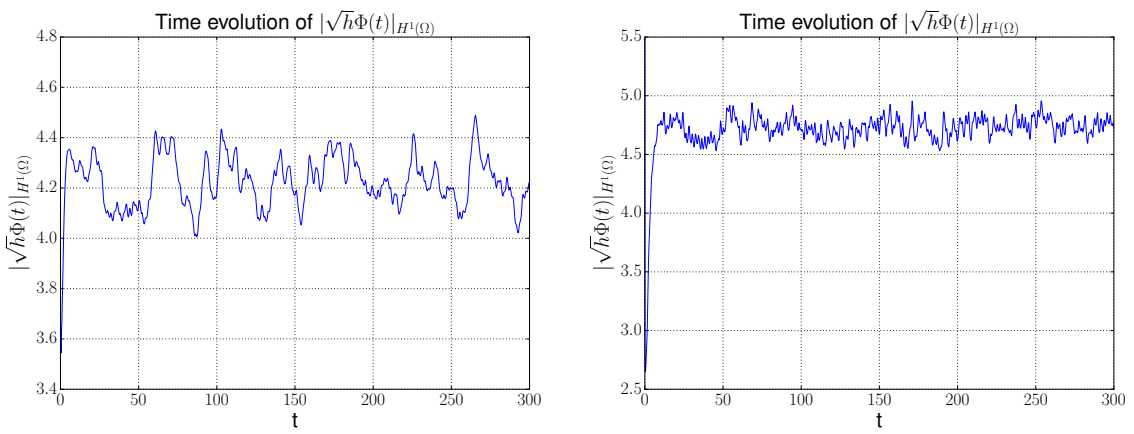

Figure 11: Time evolution of the adjoint velocity stability factor $|\sqrt{h} \hat{\varphi}|_{H^{1}(\Omega)}$ for adaptive iteration 5 (left) and iteration 15 (right). 

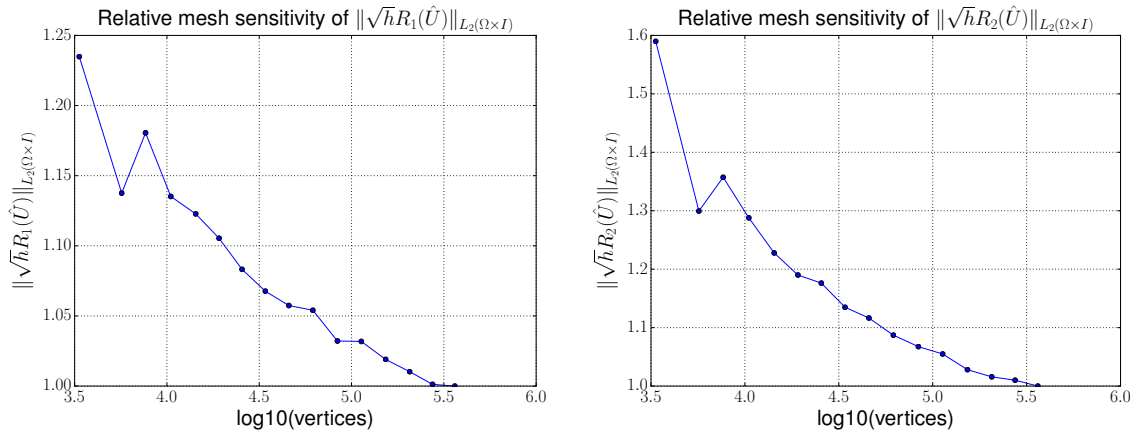

Figure 12: Mesh sensitivity of the momentum residual $\left\|\sqrt{h} R_{1}(\hat{U})\right\|_{L_{2}(\Omega \times I)}$ (left) and the continuity residual $\left\|\sqrt{h} R_{2}(\hat{U})\right\|_{L_{2}(\Omega \times I)}$ (right).
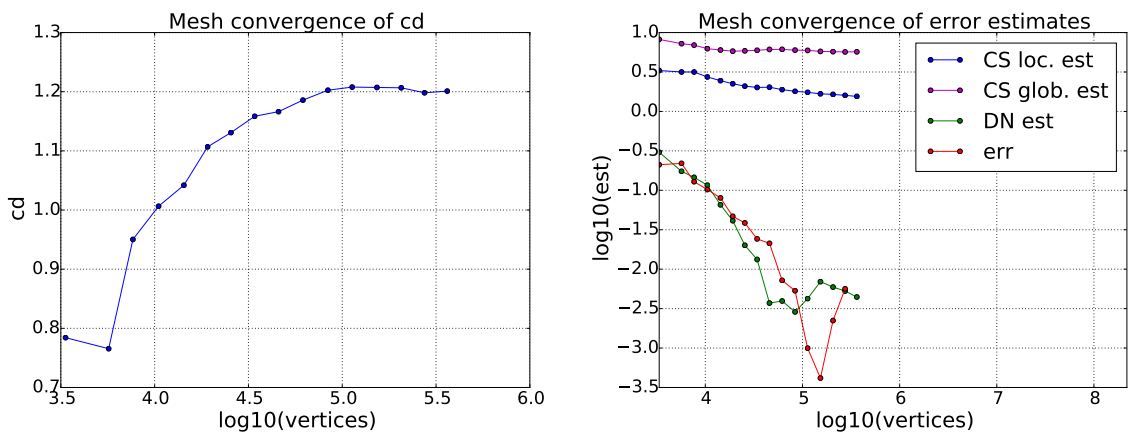

Figure 13: Mesh convergence of the drag coefficient cd (left) and error estimates (right). 


\subsection{Medium Reynolds Number Flow}

At medium Reynolds numbers, less than $R e=10^{5}$, boundary layers are laminar and can be resolved by the computational mesh. For this case, viscous effects are not negligible so that the viscosity is kept in the model, and no slip boundary conditions are chosen where the velocity is set to the same velocity as the solid boundary. DFS in the form of the cG(1)cG(1) method has been validated for a number of model problems of simple geometry bluff bodies, including a surface mounted cube and a rectangular cylinder [62, 61], a sphere [63] and a circular cylinder [45]. In each case, convergence is observed for output quantities such as drag, lift and pressure coefficients, and Strouhal numbers, and the adaptive algorithm leads to an efficient method often using orders of magnitude fewer number of degrees of freedom compared to LES methods based on ad hoc design of the mesh.

\subsection{High Reynolds Number Flow}

In high Reynolds number flow, with $R e>10^{6}$, boundary layers are turbulent and are in most cases too expensive to resolve, and must instead be modeled. To accurately predict e.g. aerodynamic forces it is critical to capture the correct flow separation, which can be connected to the boundary layer model, in case flow separation is not triggered by sharp features in the geometry [69]. Drag crisis for a circular cylinder is an illustrative example, where flow separation moves downstream the cylinder surface as a consequence of transition to turbulence in the boundary layer [74]. In [75] we model drag crisis by decreasing a skin friction parameter $\beta$, where we find that the DFS simulations reproduce the observed drag crisis scenario from experiments $[76,77,78,79]$.

In particular, we find that for small $\beta$ the solution is insensitive to the particular value of $\beta$, so that we reach an ultimate regime where the turbulent boundary layer is modeled by a zero friction free slip boundary condition. This free slip model has the benefit of being a parameter-free model of turbulent boundary layers, which requires no boundary layer mesh since no boundary layer is resolved. The basic assumptions underlying the model is that the turbulent boundary layers are fully developed, since transition to turbulence in the boundary layers is not part of the model.

To assess the capability of DFS for challenging high Reynolds number flow problems in complex geometry, we have participated in a number of benchmark workshops with detailed experimental data available for validation, including the first and second Workshop on Benchmark problems for Airframe Noise Computations (BANC-I,-II) [82, 81], and the 2nd AIAA CFD High Lift Prediction Workshop (HiLiftPW-2) [80], see Fig. 14.

Good agreement was found between DFS simulations and experimental measurements in all workshops, and two features distinguished DFS from all other methodologies: (i) DFS was the only method using adaptive mesh optimization, and (ii) DFS was the only method leaving boundary layers unresolved (free slip boundary conditions were used for all problems). Both (i) and (ii) contributed to the fact that the number of degrees 

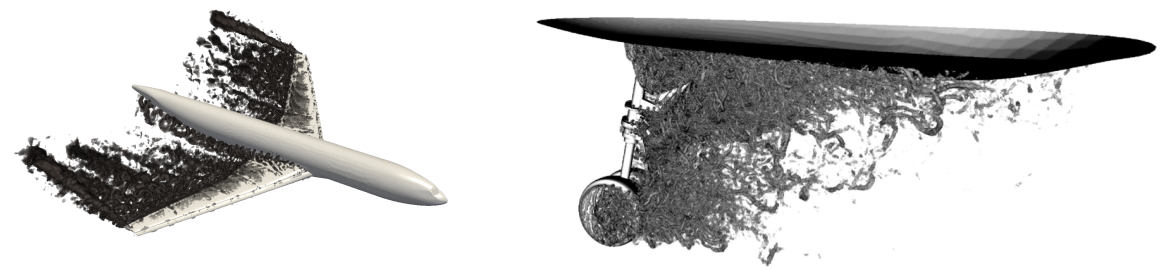

Figure 14: Vorticity visualised by a Q-criterion: snapshot of a DFS solution of the flow around of a wing-body configuration (left) [80], and flow around a Gulfstream G550 nose landing gear (right) [81].
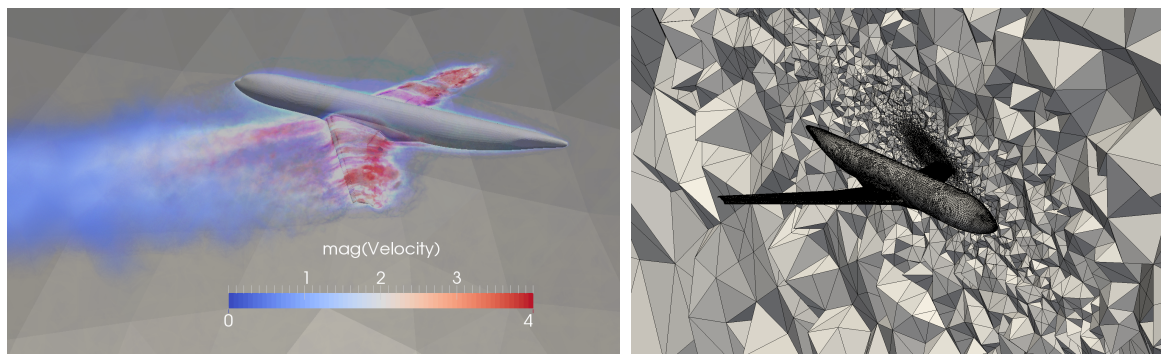

Figure 15: From [80]: Snapshot of the magnitude of dual velocity (left), and an adaptively refined computational mesh optimised for lift and drag approximation (right). 

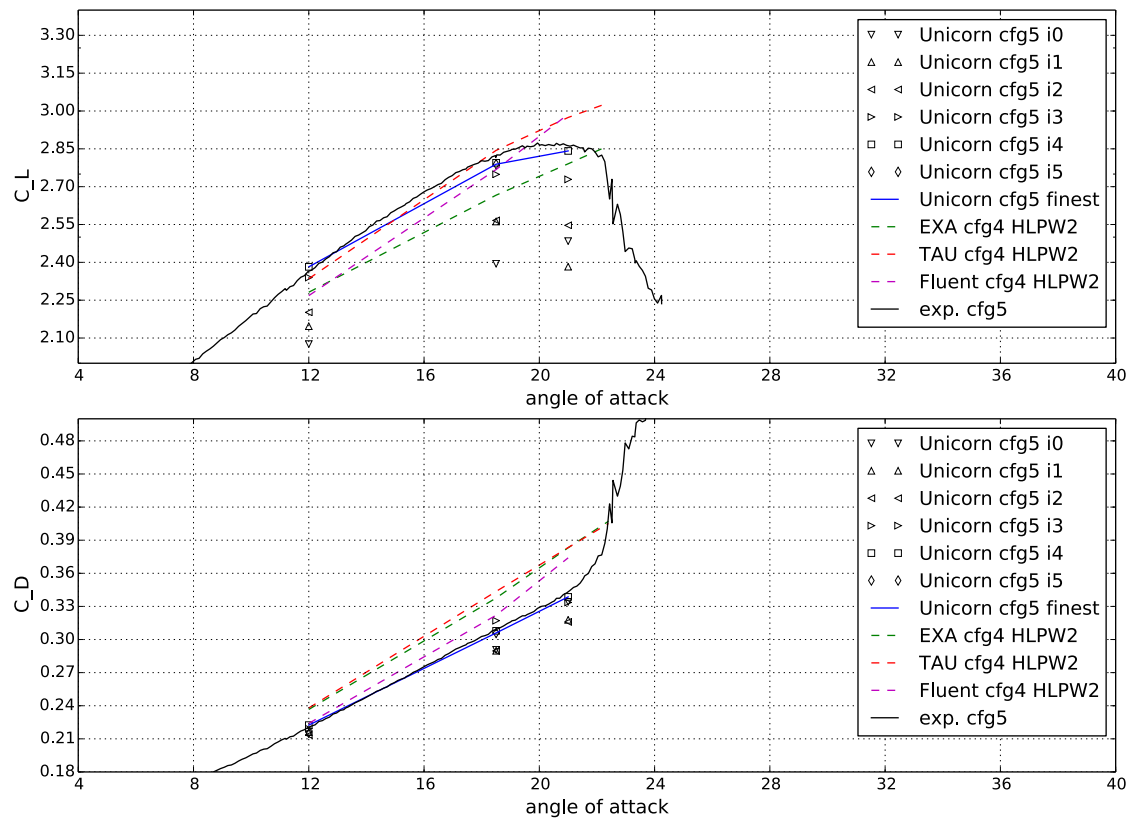

Figure 16: Convergence under adaptive mesh refinement of drag and lift coefficients towards experimental values (DFS/Unicorn) [80], compared to state-of-the-art CFD methods (EXA, TAU, Fluent) (published as part of the HighLiftPW-2 workshop). 
of freedom used in DFS was significantly lower than in other methods, often by several magnitudes, see Fig. 15 .

In HiLiftPW-2 the task was to simulate flow at high Reynolds number around a full wing-body model of an airplane, where DFS was the only method based on solving the unsteady Navier-Stokes equations [80]; aside from 2 contributions based on Lattice Boltzmann Methods all other contributions used stationary RANS.

Good agreement of DFS simulations and experimental data was found in aerodynamic forces and surface pressure distributions on the wings, and the transient simulation data provided additional data on the flow at high angles of attack modelling take-off and landing. In particular, convergence of the adaptive algorithm to experimental reference data for lift and drag was observed for angles of attack in the pre-stalled regime, see Fig. 16.

We note that since viscosity and skin friction are assumed to be negligible, the only input data to the DFS model is the geometry of the airplane, in the form of an initial coarse mesh.

\section{Acknowledgements}

This research has been supported by EU-FET grant EUNISON 308874, the European Research Council, the Swedish Foundation for Strategic Research, the Swedish Research Council, the Basque Excellence Research Center (BERC 2014-2017) program by the Basque Government, the Spanish Ministry of Economy and Competitiveness MINECO: BCAM Severo Ochoa accreditation SEV-2013-0323 and the Project of the Spanish Ministry of Economy and Competitiveness with reference MTM2013-40824. We acknowledge the Swedish National Infrastructure for Computing (SNIC) at PDC - Center for High-Performance Computing for awarding us access to the supercomputer resources Beskow. The initial volume mesh was generated with ANSA from Beta-CAE Systems S. A., who generously provided an academic license for this project.

\section{References}

[1] Didier Lucor, Johan Meyers, and Pierre Sagaut. Sensitivity analysis of large-eddy simulations to subgrid-scale-model parametric uncertainty using polynomial chaos. Journal of Fluid Mechanics, 585:255$279,2007$.

[2] Sai Hung Cheung, Todd A Oliver, Ernesto E Prudencio, Serge Prudhomme, and Robert D Moser. Bayesian uncertainty analysis with applications to turbulence modeling. Reliability Engineering 85 System Safety, 96(9):1137-1149, 2011.

[3] Eric Dow and Qiqi Wang. Quantification of structural uncertainties in the k- $\omega$ turbulence model. AIAA Paper, 1762:2011, 2011. 
[4] MJ Berger, Michael Aftosmis, and John Melton. Accuracy, adaptive methods and complex geometry. In Proc. 1st AFOSR Conf. on Dynam. Mot. in CFD, 1996.

[5] I Babuvška and Werner C Rheinboldt. Error estimates for adaptive finite element computations. SIAM Journal on Numerical Analysis, 15(4):736-754, 1978.

[6] Randolph E Bank and Bruno D Welfert. A posteriori error estimates for the stokes problem. SIAM Journal on Numerical Analysis, 28(3):591-623, 1991.

[7] Randolph E Bank and R Kent Smith. A posteriori error estimates based on hierarchical bases. SIAM Journal on Numerical Analysis, 30(4):921-935, 1993.

[8] Rüdiger Verfürth. A review of a posteriori error estimation and adaptive mesh-refinement techniques. John Wiley \& Sons Inc, 1996.

[9] Mark Ainsworth and J Tinsley Oden. A posteriori error estimation in finite element analysis. Computer Methods in Applied Mechanics and Engineering, 142(1):1-88, 1997.

[10] I Babuška and A Miller. The post-processing approach in the finite element methodpart 3: A posteriori error estimates and adaptive mesh selection. International Journal for Numerical Methods in Engineering, 20(12):2311-2324, 1984.

[11] Kenneth Eriksson and Claes Johnson. An adaptive finite element method for linear elliptic problems. Mathematics of Computation, 50(182):361-383, 1988.

[12] Roland Becker and Rolf Rannacher. A feed-back approach to error control in finite element methods: Basic analysis and examples. Citeseer, 1996.

[13] Paul Houston, Rolf Rannacher, and Endre Süli. A posteriori error analysis for stabilised finite element approximations of transport problems. Computer methods in applied mechanics and engineering, 190(11):1483-1508, 2000.

[14] AT Patera and J Peraire. A general lagrangian formulation for the computation of a-posteriori finite element bounds. chapter in error estimation and adaptive discretization methods in cfd. Lecture notes in computational science and engineering, 25, 2002.

[15] Randolph E Bank and Michael Holst. A new paradigm for parallel adaptive meshing algorithms. SIAM review, 45(2):291-323, 2003.

[16] Kenneth Eriksson, Don Estep, Peter Hansbo, and Claes Johnson. Introduction to adaptive methods for differential equations. Acta numerica, 4(1):105-158, 1995.

[17] Roland Becker and Rolf Rannacher. An optimal control approach to a posteriori error estimation in finite element methods. Acta Numerica 2001, 10:1-102, 2001.

[18] Michael B Giles and Endre Süli. Adjoint methods for pdes: a posteriori error analysis and postprocessing by duality. Acta Numerica, 11:145-236, 2002. 
[19] Claes Johnson, Rolf Rannacher, and Mats Boman. Numerics and hydrodynamic stability: toward error control in computational fluid dynamics. SIAM Journal on Numerical Analysis, 32(4):1058-1079, 1995.

[20] Michael B Giles, M Larson, M Levenstam, and Endre Suli. Adaptive error control for finite element approximations of the lift and drag coefficients in viscous flow. Technical Report, Oxford University, 1997.

[21] S Prudhomme and JT Oden. Numerical stability and error analysis for the incompressible navier-stokes equations. Communications in numerical methods in engineering, 18(11):779-787, 2002.

[22] Johan Hoffman and Claes Johnson. Adaptive finite element methods for incompressible fluid flow. In Error estimation and adaptive discretization methods in computational fluid dynamics, pages 97-157. Springer, 2003.

[23] Malte Braack and Thomas Richter. Solutions of 3d navier-stokes benchmark problems with adaptive finite elements. Computers $\&$ fluids, 35(4):372-392, 2006.

[24] Claes Johnson. Adaptive finite element methods for conservation laws. In Advanced numerical approximation of nonlinear hyperbolic equations, pages 269-323. Springer, 1998.

[25] Robert Sandboge. Adaptive finite element methods for systems of reaction-diffusion equations. Computer methods in applied mechanics and engineering, 166(3):309-328, 1998.

[26] Erik Burman. Adaptive finite element methods for compressible twophase flow. Mathematical Models and Methods in Applied Sciences, 10(07):963-989, 2000.

[27] Erik Burman. Adaptive finite element methods for compressible flow. Computer methods in applied mechanics and engineering, 190(8):1137-1162, 2000.

[28] Ralf Hartmann and Paul Houston. Adaptive discontinuous galerkin finite element methods for the compressible euler equations. Journal of Computational Physics, 183(2):508-532, 2002.

[29] TJ Barth. Numerical methods and error estimation for conservation laws on structured and unstructured meshes. Lecture Notes, Series, 4, 2003.

[30] Erik Burman, Alexandre Ern, and Vincent Giovangigli. An adaptive finite element method with crosswind diffusion for low mach, steady, laminar combustion. Journal of Computational Physics, 188(2):472492, 2003.

[31] Timothy J Barth. Space-time error representation and estimation in navier-stokes calculations. In Complex Effects in Large Eddy Simulations, pages 29-48. Springer, 2007.

[32] Ethan J Kubatko, Shintaro Bunya, Clint Dawson, and Joannes J Westerink. Dynamic p-adaptive runge-kutta discontinuous galerkin methods for the shallow water equations. Computer Methods in Applied Mechanics and Engineering, 198(21):1766-1774, 2009. 
[33] Paul Houston, Christoph Schwab, and Endre Süli. Stabilized hpfinite element methods for first-order hyperbolic problems. SIAM Journal on Numerical Analysis, 37(5):1618-1643, 2000.

[34] Vincent Heuveline and Rolf Rannacher. Duality-based adaptivity in the hp-finite element method. Journal of Numerical Mathematics jnma, 11(2):95-113, 2003.

[35] Stefano Giani and Paul Houston. Anisotropic hp-adaptive discontinuous galerkin finite element methods for compressible fluid flows. International Journal of Numerical Analysis and Modeling, 2011.

[36] MJ Castro-Diaz, F Hecht, B Mohammadi, and O Pironneau. Anisotropic unstructured mesh adaption for flow simulations. International Journal for Numerical Methods in Fluids, 25(4):475-491, 1997.

[37] David A Venditti and David L Darmofal. Anisotropic grid adaptation for functional outputs: application to two-dimensional viscous flows. Journal of Computational Physics, 187(1):22-46, 2003.

[38] EM Lee-Rausch, MA Park, WT Jones, DP Hammond, and EJ Nielsen. Application of parallel adjoint-based error estimation and anisotropic grid adaptation for three-dimensional aerospace configurations. AIAA paper, 4842:2005, 2005.

[39] R Schneider and PK Jimack. Toward anisotropic mesh adaption based upon sensitivity of a posteriori estimates. Doctoral thesis, University of Leeds, 2005.

[40] MD Piggott, PE Farrell, CR Wilson, GJ Gorman, and CC Pain. Anisotropic mesh adaptivity for multi-scale ocean modelling. Philosophical Transactions of the Royal Society of London A: Mathematical, Physical and Engineering Sciences, 367(1907):4591-4611, 2009.

[41] Tobias Leicht and Ralf Hartmann. Error estimation and anisotropic mesh refinement for 3d laminar aerodynamic flow simulations. Journal of Computational Physics, 229(19):7344-7360, 2010.

[42] Thierry Coupez and Elie Hachem. Solution of high-reynolds incompressible flow with stabilized finite element and adaptive anisotropic meshing. Computer Methods in Applied Mechanics and Engineering, 267:65-85, 2013.

[43] Stefano Micheletti, Simona Perotto, and Filippo David. Model adaptation enriched with an anisotropic mesh spacing for nonlinear equations: application to environmental and cfd problems. Numerical Mathematics: Theory, Methods and Applications, 6(03):447-478, 2013.

[44] Qiqi Wang and Jun-Hui Gao. The drag-adjoint field of a circular cylinder wake at reynolds numbers 20, 100 and 500. Journal of Fluid Mechanics, 730:145-161, 2013.

[45] Johan Hoffman. Efficient computation of mean drag for the subcritical flow past a circular cylinder using general galerkin g2. International journal for numerical methods in fluids, 59(11):1241-1258, 2009 . 
[46] Murtazo Nazarov and Johan Hoffman. On the stability of the dual problem for high reynolds number flow past a circular cylinder in two dimensions. SIAM Journal on Scientific Computing, 34(4):A1905A1924, 2012.

[47] Johan Hoffman and Claes Johnson. Computational Turbulent Incompressible Flow: Applied Mathematics: Body and Soul 4, volume 4. Springer, 2007.

[48] Ralf Hartmann, Joachim Held, and Tobias Leicht. Adjoint-based error estimation and adaptive mesh refinement for the rans and k$\omega$ turbulence model equations. Journal of Computational Physics, 230(11):4268-4284, 2011.

[49] Johan Hoffman. On duality-based a posteriori error estimation in various norms and linear functionals for large eddy simulation. SIAM Journal on Scientific Computing, 26(1):178-195, 2004.

[50] Adrian Dunca, Volker John, and William Layton. Approximating local averages of fluid velocities: The equilibrium navier-stokes equations. Applied numerical mathematics, 49(2):187-205, 2004.

[51] Johan Hoffman. Dynamic subgrid modelling for time dependent convection-diffusion-reaction equations with fractal solutions. International journal for numerical methods in fluids, 40(3-4):583-592, 2002.

[52] Johan Hoffman. Subgrid modeling for convection-diffusion-reaction in two space dimensions using a haar multiresolution analysis. Mathematical Models and Methods in Applied Sciences, 13(10):1515-1536, 2003.

[53] Johan Hoffman, Claes Johnson, and Silvia Bertoluzza. Subgrid modeling for convection-diffusion-reaction in one space dimension using a haar multiresolution analysis. Computer methods in applied mechanics and engineering, 194(1):19-44, 2005.

[54] Markus Klein, Johan Meyers, and Bernard J Geurts. Assessment of les quality measures using the error landscape approach. In Quality and Reliability of Large-Eddy Simulations, pages 131-142. Springer, 2008.

[55] Johan Meyers, Bernard Geurts, and Pierre (Eds.) Sagaut. Quality and Reliability of Large-Eddy Simulations. ERCOFTAC Series, 2008.

[56] M.V. Salvetti, B. Geurts, J. Meyers, and P. (Eds.) Sagaut. Quality and Reliability of Large-Eddy Simulations II. ERCOFTAC Series, 2011.

[57] John VonNeumann and Robert D Richtmyer. A method for the numerical calculation of hydrodynamic shocks. Journal of applied physics, 21(3):232-237, 1950.

[58] Joseph Smagorinsky. General circulation experiments with the primitive equations: I. the basic experiment*. Monthly weather review, 91(3):99-164, 1963. 
[59] Y Bazilevs, VM Calo, JA Cottrell, TJR Hughes, A Reali, and G Scovazzi. Variational multiscale residual-based turbulence modeling for large eddy simulation of incompressible flows. Computer Methods in Applied Mechanics and Engineering, 197(1):173-201, 2007.

[60] Fernando F Grinstein, Len G Margolin, and William J Rider. Implicit large eddy simulation: computing turbulent fluid dynamics. Cambridge university press, 2007.

[61] Johan Hoffman and Claes Johnson. A new approach to computational turbulence modeling. Computer Methods in Applied Mechanics and Engineering, 195(23):2865-2880, 2006.

[62] Johan Hoffman. Computation of mean drag for bluff body problems using adaptive dns/les. SIAM Journal on Scientific Computing, 27(1):184-207, 2005.

[63] Johan Hoffman. Adaptive simulation of the subcritical flow past a sphere. Journal of Fluid Mechanics, 568:77-88, 2006.

[64] Murtazo Nazarov and Johan Hoffman. Residual-based artificial viscosity for simulation of turbulent compressible flow using adaptive finite element methods. International Journal for Numerical Methods in Fluids, 71(3):339-357, 2013.

[65] Rodrigo Vilela Abreu, Niclas Jansson, and Johan Hoffman. Adaptive computation of aeroacoustic sources for a rudimentary landing gear. International Journal for Numerical Methods in Fluids, 74(6):406421, 2014.

[66] Johan Hoffman, Johan Jansson, Niclas Jansson, and Rodrigo Vilela De Abreu. Towards a parameter-free method for high reynolds number turbulent flow simulation based on adaptive finite element approximation. Computer Methods in Applied Mechanics and Engineering, 288:60-74, 2015.

[67] Johan Hoffman and Claes Johnson. Blow up of incompressible euler solutions. BIT Numerical Mathematics, 48(2), 2008.

[68] Johan Hoffman. Adaptive turbulence computation based on weak solutions and weak uniqueness. In Quality and Reliability of LargeEddy Simulations, pages 21-36. Springer, 2008.

[69] Johan Hoffman, Johan Jansson, and Rodrigo Vilela De Abreu. Adaptive modeling of turbulent flow with residual based turbulent kinetic energy dissipation. Computer Methods in Applied Mechanics and Engineering, 200(37):2758-2767, 2011.

[70] Jean Leray. Sur le mouvement d'un liquide visqueux emplissant l'espace. Acta mathematica, 63(1):193-248, 1934.

[71] Johan Hoffman, Johan Jansson, Rodrigo Vilela de Abreu, Niyazi Cem Degirmenci, Niclas Jansson, Kaspar Müller, Murtazo Nazarov, and Jeannette Hiromi Spühler. Unicorn: Parallel adaptive finite element simulation of turbulent flow and fluid-structure interaction for deforming domains and complex geometry. Computers \&s Fluids, 80:310-319, 2013. 
[72] Anders Logg, Kent-Andre Mardal, Garth N. Wells, et al. Automated Solution of Differential Equations by the Finite Element Method. Springer, 2012.

[73] Johan Hoffman, Johan Jansson, and Niclas Jansson. Fenics-hpc: Automated predictive high-performance finite element computing with applications in aerodynamics. Proceedings of the 11th International Conference on Parallel Processing and Applied Mathematics, PPAM 2015. Lecture Notes in Computer Science, 2015.

[74] MM Zdravkovich. Flow around circular cylinders, vol. 1. fundamentals. Journal of Fluid Mechanics, 350:377-378, 1997.

[75] Johan Hoffman and Niclas Jansson. A computational study of turbulent flow separation for a circular cylinder using skin friction boundary conditions. In Quality and Reliability of Large-Eddy Simulations II, pages 57-68. Springer, 2011.

[76] AI Korotkin. The three-dimensional character of a cross flow around a circular cylinder. TsAGI, Uchenye Zapiski, 4(5):26-33, 1973.

[77] John S Humphreys. On a circular cylinder in a steady wind at transition reynolds numbers. Journal of Fluid Mechanics, 9(04):603-612, 1960.

[78] Burkhard Gölling. Experimentelle Untersuchungen des laminarturbulenten Überganges der Zylindergrenzschichtströmung. $\mathrm{PhD}$ thesis, DLR, 2001.

[79] Günter Schewe. Reynolds-number effects in flow around more-or-less bluff bodies. Journal of Wind Engineering and Industrial Aerodynamics, 89(14):1267-1289, 2001.

[80] Johan Hoffman, Johan Jansson, Niclas Jansson, and Rodrigo Vilela de Abreu. Time-resolved adaptive fem simulation of the dlr-f11 aircraft model at high reynolds number. In AIAA 2014-0917, Proc. 52nd Aerospace Sciences Meeting, AIAA SciTech, 2014.

[81] R Vilela de Abreu, N Jansson, and J Hoffman. Computation of aeroacoustic sources for a complex nose landing gear geometry using adaptivity. In Proceedings of the Second Workshop on Benchmark problems for Airframe Noise Computations (BANC-II), Colorado Springs, 2012.

[82] Rodrigo Vilela de Abreu, Niclas Jansson, and Johan Hoffman. Adaptive computation of aeroacoustic sources for a rudimentary landing gear using lighthills analogy. AIAA Paper, 2942:2011, 2011. 